\title{
A KINEMATICALLY SELECTED, METAL-POOR STELLAR HALO IN THE OUTSKIRTS OF M31
}

\author{
S. C. Chapman, ${ }^{1}$ R. Ibata, ${ }^{2}$ G. F. Lewis, ${ }^{3}$ A. M. N. Ferguson, ${ }^{4}$ M. Irwin, ${ }^{5}$ A. McConnachie, ${ }^{6}$ and N. Tanvir ${ }^{7}$ \\ Received 2006 February 26; accepted 2006 August 15
}

\begin{abstract}
We present evidence for a metal-poor, $[\mathrm{Fe} / \mathrm{H}] \sim-1.4, \sigma=0.2 \mathrm{dex}$, stellar halo component detectable at radii from 10 to $70 \mathrm{kpc}$, in our nearest giant spiral neighbor, the Andromeda galaxy. This metal-poor sample underlies the recently discovered extended rotating component and has no detected metallicity gradient. This discovery uses a large sample of 9861 radial velocities of red giant branch (RGB) stars obtained with the Keck II Telescope and DEIMOS spectrograph, with 827 stars with robust radial velocity measurements isolated kinematically to lie in the halo component primarily by windowing out the extended rotating component, which dominates the photometric profile of Andromeda out to $<50 \mathrm{kpc}$ (deprojected). The stars lie in 54 spectroscopic fields spread over an $8 \mathrm{deg}^{2}$ region, and are expected to fairly sample the halo to a radius of $\sim 70 \mathrm{kpc}$. The halo sample shows no significant evidence for rotation. Fitting a simple model in which the velocity dispersion of the component decreases with radius, we find a central velocity dispersion of $152 \mathrm{~km} \mathrm{~s}^{-1}$ decreasing by $-0.90 \mathrm{~km} \mathrm{~s}^{-1} \mathrm{kpc}^{-1}$. By fitting a cosmologically motivated NFW halo model to the halo stars we constrain the virial mass of M31 to be greater than $9.0 \times 10^{11} M_{\odot}$ with $99 \%$ confidence. The properties of this halo component are very similar to that found in our Milky Way, revealing that these roughly equal mass galaxies may have led similar accretion and evolutionary paths in the early universe.
\end{abstract}

Subject headings: galaxies: evolution — galaxies: individual (Andromeda) — galaxies: spiral — Local Group

Online material: color figures

\section{INTRODUCTION}

One of the fundamental tenets of the cold dark matter (CDM) paradigm (White \& Rees 1978) is that dark matter halos form hierarchically, via a series of mergers with smaller halos. This prediction gives rise to the natural expectation that the stellar halo ${ }^{8}$ is formed from disrupted, accreted dwarf galaxies (Johnston et al. 1999; Helmi \& White 1999a; Bullock et al. 2001; Bullock \& Johnston 2004).

CDM models predict that stellar halos form early, with the majority of the stars in the halo formed within a few relatively massive, $5 \times 10^{10} M_{\odot}$, dwarf irregular (dIrr) sized dark matter halos. These were accreted $\sim 10$ Gyr in the past, likely prior to the formation of the bulge component via gaseous collapse, mergers, and starbursts. Such models predict that the halo should be metalpoor and relatively smooth within the inner $50 \mathrm{kpc}$, where the tidal signatures of early stellar streams have been erased (Bullock \& Johnston 2005). The giant stellar streams in the Milky Way (Ibata et al. 1994, 2001b; Newberg et al. 2002) and Andromeda (Ibata et al. 2001a; McConnachie et al. 2004) testify to the longevity of minor accretions that occurred since the metal poor halo formed. The average stellar halo density profile is expected to fall off with radius more quickly than that of the dark matter because the stellar halo is formed from the most tightly bound material in

\footnotetext{
${ }^{1}$ California Institute of Technology, Pasadena, CA 91125; schapman@astro caltech.edu.

2 Observatoire de Strasbourg, 11, rue de l'Université, F-67000 Strasbourg, France.

3 Institute of Astronomy, School of Physics, A29, University of Sydney, NSW 2006, Australia.

${ }^{4}$ Institute for Astronomy, University of Edinburgh, Edinburgh EH19 3HJ, $\mathrm{UK}$.

5 Institute of Astronomy, Madingley Road, Cambridge CB3 0HA, UK.

${ }^{6}$ University of Victoria, Department of Physics and Astronomy, Victoria, BC, V8P 1A1, Canada.

7 Physical Sciences, University of Hertfordshire, Hatfield AL10 9AB, UK.

8 We use "halo" to mean "related to dark halo," and typically use "stellar halo" for the stellar component that partially fills the dark halo.
}

infalling systems, while the majority of the accreted dark matter is stripped and deposited at larger radii. The discovery of fossil evidence of these accretion events via the identification of substructure within nearby stellar halos may provide the only direct evidence that structure formation is hierarchical on small scales (Bullock \& Johnston 2005).

One of the most promising systems for the detailed decomposition and study of the stellar halo and its relation to the dark matter halo is the Andromeda galaxy, or M31 (Walterbos \& Kennicutt 1988; Pritchet \& van den Bergh 1988; Irwin et al. 2005; Geehan et al. 2006; Gilbert et al. 2006). Often considered the Milky Way's twin, this galaxy, at a distance of $\sim 785 \mathrm{kpc}$, (McConnachie et al. 2005), lies sufficiently close that modern instrumentation can be used to measure spectra of individual stars in the top few magnitudes of the red giant branch (RGB), providing access to their radial velocities and metallicities. In this paper we probe the outer stellar component of M31 using radial velocities and $\mathrm{Ca}$ II triplet $(\mathrm{CaT})$ metallicities of RGB stars to study the overall kinematic characteristics.

\section{OBSERVATIONS AND SAMPLE DEFINITION}

Since 2002 we have undertaken a spectroscopic survey of RGB stars (selected by their optical colors and magnitudes) in M31 with the $10 \mathrm{~m} \mathrm{W.} \mathrm{M.} \mathrm{Keck} \mathrm{II} \mathrm{Telescope} \mathrm{and} \mathrm{the} \mathrm{DEIMOS}$ spectrograph (Faber et al. 2003). The 54 spectroscopic fields shown in Figure 1 comprise 9861 stars with measured radial velocities (shown in Fig. 2), and represent our ongoing program to provide a complete kinematic and metallicity characterization of the disk and halo of M31, as well as the accreted fragments that now litter this environment. Details of the spectroscopic observations and the selection of M31 RGB stars from the colormagnitude diagram (CMD) have been presented in previous papers ( Ibata et al. 2005, hereafter I05; McConnachie et al. 2003; Ferguson et al. 2002). Briefly, we used the standard DEIMOS multislit mode for low-density outer fields, but adopted our own minislitlets approach targeting $>600$ stars per mask in higher 


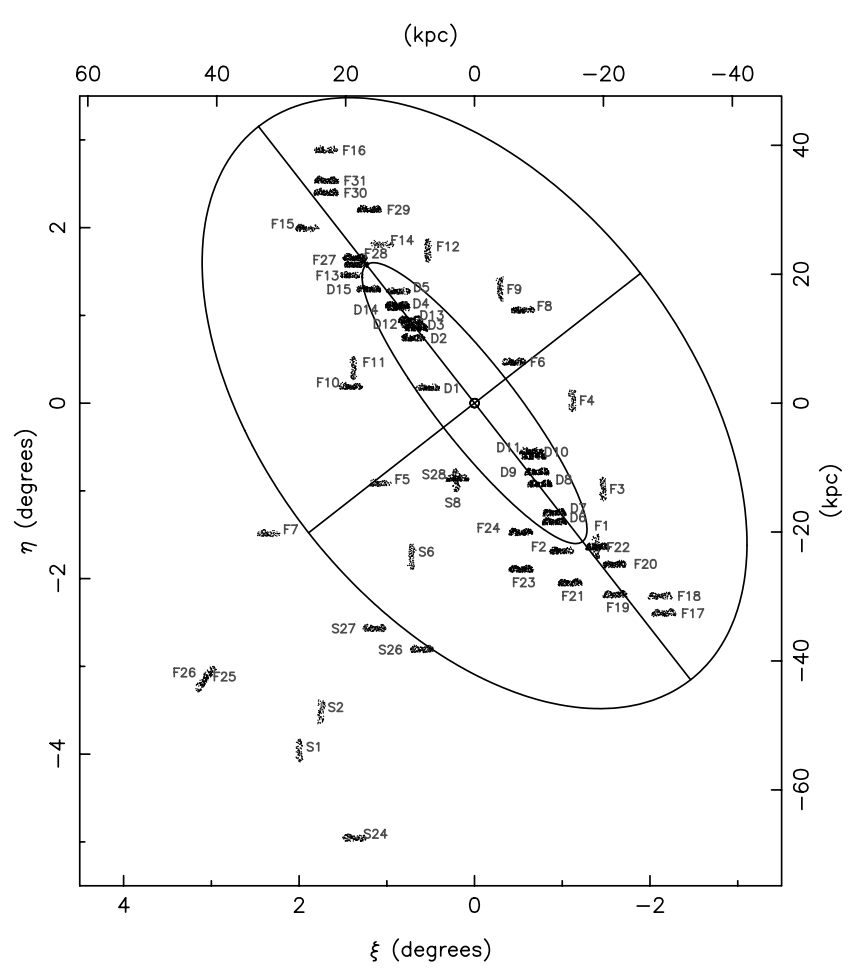

FIG. 1.-Current coverage of our radial velocity survey of M31 with the DEIMOS spectrograph, in standard coordinates $(\chi, \eta)$. The outer ellipse shows a segment of a $55 \mathrm{kpc}$ radius ellipse flattened to $c / a=0.6$, and the major and minor axis are indicated with straight lines out to this ellipse. The inner ellipse corresponds to a disk of radius $2^{\circ}(27 \mathrm{kpc})$, with the same inclination as the galactic disk. The distribution of 54 Keck/DEIMOS fields, comprising 9861 stars with measured radial velocities, are overlaid on the survey region, with field names tied to Table 1. [See the electronic edition of the Journal for a color version of this figure.]

density inner fields using small (1".5 long) slits, with data reduction using our own custom software pipeline (I05). Stars are selected for masks based on the position of the RGB in the color-magnitude diagram, with a limit of $I<22$ for useful spectra. For most fields, except where a structure like And IX (Chapman et al. 2005) or the Huxor et al. (2005) fuzzy clusters were targeted, stars were given equal priority from the RGB tip to $I=$ 21.25, then lower weight for fainter objects. The signal-to-noise ratio $(\mathrm{S} / \mathrm{N})$ degrades to about 5 at the limit of useful data. A summary of all fields observed with DEIMOS, the radial velocity yields, and the breakdown of stars by kinematics into halo, disk, and Galactic windows, is presented in Table 1. Representative individual spectra are shown in Figure 3, as a function of both RGB color and $I$ mag.

Radial velocities measured from the $\mathrm{Ca}$ II triplet $(\mathrm{CaT})$ can be used to kinematically isolate a likely sample of M31 halo stars. However, extracting a representative sample of M31 halo stars is hampered by the fact that many of the spectroscopic pointings have directly targeted the obvious photometric overdensities identified in Ferguson et al. (2002). Fortunately, our discovery (I05) that an extended rotating component dominates the star counts in all these photometric substructures (with the exception of the giant tidal stream to the southeast; Ibata et al. 2001a, 2004; McConnachie et al. 2003; Chapman et al. 2006; Guhathakurta et al. 2006; Kalirai et al. 2006a), suggests a method to procure a sample of stars that is unbiased with respect to the photometric overdensities. A candidate halo component of stars can be extracted by windowing out in heliocentric velocity all stars that rotate with this extended component (shown in

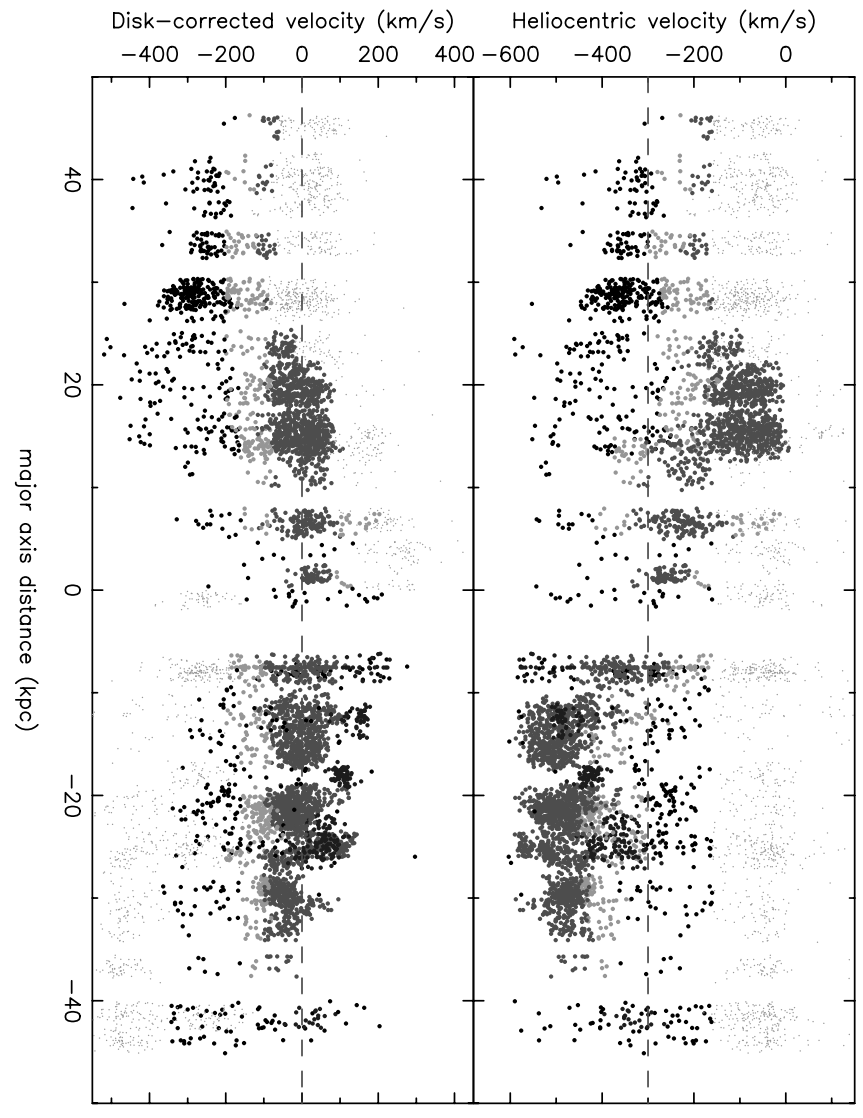

FIG. 2.- Radial velocity measurements from our 54 spectroscopic fields are displayed as a function of distance along the major axis, with the top panel showing heliocentric values, and the bottom panel velocities corrected for the expected motion of the disk (using the model of I05). The foreground Milky Way stars (small faint gray dots) are removed from our sample. The remaining stars are divided into a disklike population (disk-corrected velocity close to zero; medium dark gray dots), an intermediate thick-disk-like population (light gray dots), and a halo-like population (a broad component which is not rotating with the disk; black dots), and those metal-rich stars identified with the giant southern stream (dark gray dots). [See the electronic edition of the Journal for a color version of this figure.]

Fig. 2). ${ }^{9}$ In other words, the photometric representation of our windowed sample should be spatially smooth to first order (see I05 for further discussion of this point). We construct windows for the extended rotating component by adopting the average fit to the extended rotating component, $\sigma_{v}=50 \mathrm{~km} \mathrm{~s}^{-1}$ Gaussian, from the kinematic model presented in I05, and considering the window to span $\pm 2 \sigma$ (or $\pm 100 \mathrm{~km} \mathrm{~s}^{-1}$ in the disk-lag frame). ${ }^{10}$ This is considered to be our "disk" sample, those stars exhibiting thin-disk-like kinematics. Between disk-lag velocities of $-160<$ $v_{\text {lag }}<-100 \mathrm{~km} \mathrm{~s}^{-1}$ we define an additional component of intermediate kinematics, which we term "thick disk" in the subsequent discussion (to be discussed in detail in a subsequent paper). As the peak of the velocity distribution of the disk is observed to shift slightly between fields (typically by $20-30 \mathrm{~km} \mathrm{~s}^{-1}$ ) compared to the simple disk model of I05, the selection windows are

\footnotetext{
9 Note that a recnt theory of the extended disk formation by Penarrubia et al. (2006) suggests a dwarf accretion origin, which would make this roating component a viable component of the stellar halo, albeit one that formed much more recently than the primordial halo discussed in this paper.

10 While the dispersion of the disklike component is about $30 \mathrm{~km} \mathrm{~s}^{-1}$ on average in individual fields (I05), it is broader $\left(\sim 50 \mathrm{~km} \mathrm{~s}^{-1}\right)$ in the co-added disk fields. This is partly a problem of the projection effects and the simplicity of the kinematic model currently adopted.
} 
TABLE 1

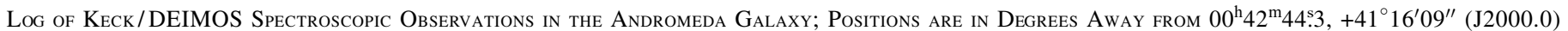

\begin{tabular}{|c|c|c|c|c|c|c|c|c|c|}
\hline Number & Field & Name & $\chi$ & $\eta$ & No. of Stars ${ }^{\mathrm{a}}$ & No. of Halos ${ }^{b}$ & No. of Disks ${ }^{\mathrm{c}}$ & No. of Galaxies ${ }^{\mathrm{d}}$ & Observation Run \\
\hline $1 \ldots \ldots \ldots \ldots \ldots \ldots \ldots \ldots \ldots$ & $\mathrm{F} 1$ & w11 & -1.398 & -1.670 & 108 & 17 & 76 & 15 & 2002 Sep \\
\hline 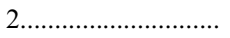 & $\mathrm{F} 2$ & 50Disk & -0.968 & -1.682 & 266 & 26 & 184 & 56 & 2004 Sep \\
\hline 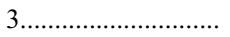 & F3 & w72 & -1.463 & -0.989 & 95 & 6 & 72 & 17 & 2002 Sep \\
\hline $4 \ldots \ldots \ldots \ldots \ldots \ldots \ldots \ldots \ldots$ & $\mathrm{F} 4$ & w80 & -1.120 & 0.020 & 79 & 6 & 49 & 24 & 2002 Sep \\
\hline $5 \ldots \ldots \ldots \ldots \ldots \ldots \ldots \ldots \ldots$ & F5 & 57Halo & 1.087 & -0.919 & 113 & 6 & 24 & 83 & 2004 Sep \\
\hline 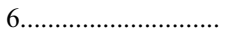 & F6 & 10Disk & -0.443 & 0.462 & 226 & 4 & 146 & 76 & 2004 Sep \\
\hline 7 & F7 & 59Halo & 2.364 & -1.488 & 96 & 3 & 13 & 80 & 2004 Sep \\
\hline 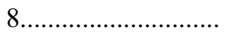 & F8 & 54Blob & -0.532 & 1.061 & 206 & 8 & 113 & 85 & 2004 Sep \\
\hline 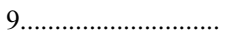 & F9 & w91 & -0.297 & 1.288 & 96 & 10 & 60 & 26 & 2002 Sep \\
\hline 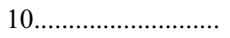 & F10 & 56Blob & 1.398 & 0.187 & 191 & 12 & 97 & 82 & 2004 Sep \\
\hline $11 \ldots \ldots \ldots \ldots \ldots \ldots \ldots \ldots \ldots$ & F11 & w42 & 1.380 & 0.395 & 333 & 85 & 49 & 199 & 2002 Sep \\
\hline 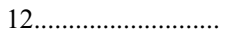 & F12 & w95 & 0.530 & 1.713 & 122 & 20 & 38 & 64 & 2002 Sep \\
\hline $13 \ldots \ldots \ldots \ldots \ldots \ldots \ldots \ldots$ & F13 & 06Disk & 1.392 & 1.453 & 176 & 57 & 13 & 106 & 2003 Sep \\
\hline $14 \ldots \ldots \ldots \ldots \ldots \ldots \ldots \ldots \ldots$ & F14 & 14Disk & 1.041 & 1.807 & 76 & 9 & 2 & 65 & 2004 Sep \\
\hline $15 \ldots \ldots \ldots \ldots \ldots \ldots \ldots \ldots \ldots \ldots \ldots \ldots \ldots \ldots$ & F15 & 53Blob & 1.943 & 1.997 & 156 & 34 & 16 & 106 & 2004 Sep \\
\hline 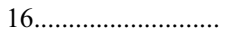 & F16 & 55Blob & 1.716 & 2.881 & 151 & 2 & 14 & 135 & 2004 Sep \\
\hline $17 \ldots \ldots \ldots \ldots$ & D1 & 17Disk & 0.518 & 0.173 & 175 & 12 & 112 & 51 & 2004 Sep \\
\hline $18 \ldots \ldots \ldots \ldots \ldots \ldots \ldots \ldots \ldots$ & D2 & 18Disk & 0.693 & 0.739 & 203 & 22 & 12 & 169 & 2004 Sep \\
\hline $19 \ldots \ldots \ldots \ldots \ldots \ldots \ldots \ldots \ldots$ & D3 & 52Disk & 0.715 & 0.896 & 207 & 19 & 17 & 171 & 2004 Sep \\
\hline $20 \ldots \ldots \ldots \ldots \ldots \ldots \ldots \ldots \ldots$ & D4 & 51Disk & 0.879 & 1.085 & 190 & 19 & 22 & 149 & 2004 Sep \\
\hline $21 \ldots \ldots \ldots \ldots \ldots \ldots \ldots \ldots$ & D5 & 04Disk & 0.862 & 1.272 & 204 & 22 & 19 & 163 & 2003 Sep \\
\hline $22 \ldots \ldots \ldots \ldots \ldots \ldots \ldots$ & $\mathrm{S} 1$ & s01 & 1.993 & -3.965 & 74 & 6 & 32 & 36 & 2002 Sep \\
\hline $23 \ldots \ldots \ldots \ldots \ldots \ldots \ldots \ldots$ & $\mathrm{S} 2$ & $\mathrm{~s} 02$ & 1.740 & -3.524 & 74 & 3 & 44 & 27 & 2002 Sep \\
\hline $24 \ldots \ldots \ldots \ldots \ldots \ldots \ldots \ldots$ & S6 & s06 & 0.712 & -1.755 & 89 & 27 & 44 & 18 & 2002 Sep \\
\hline $25 \ldots \ldots \ldots \ldots \ldots \ldots \ldots \ldots \ldots$ & S8 & $\mathrm{s} 08$ & 0.189 & -0.945 & 187 & 69 & 83 & 35 & 2002 Sep \\
\hline 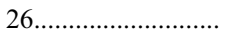 & $\mathrm{S} 24$ & s24 & 1.370 & -4.954 & 131 & 5 & 35 & 91 & 2003 Sep \\
\hline $27 \ldots \ldots \ldots \ldots \ldots \ldots \ldots \ldots$ & $\mathrm{S} 26$ & s26 & 0.593 & -2.809 & 147 & 5 & 64 & 78 & 2003 Sep \\
\hline $28 \ldots \ldots \ldots \ldots \ldots \ldots \ldots \ldots$ & $\mathrm{S} 27$ & s27 & 1.133 & -2.566 & 145 & 4 & 77 & 64 & 2003 Sep \\
\hline $29 \ldots \ldots \ldots \ldots \ldots \ldots \ldots \ldots \ldots \ldots \ldots \ldots$ & D6 & 101DiH & -0.903 & -1.353 & 279 & 12 & 257 & 10 & 2004 Sep \\
\hline 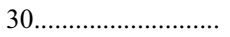 & D7 & 102DiH & -0.903 & -1.243 & 270 & 13 & 233 & 24 & 2004 Sep \\
\hline 31.......................... & D8 & 104DiH & -0.765 & -0.923 & 273 & 14 & 218 & 41 & 2004 Sep \\
\hline $32 \ldots \ldots \ldots \ldots \ldots \ldots \ldots \ldots \ldots$ & D9 & 105DiH & -0.716 & -0.786 & 273 & 13 & 244 & 16 & 2004 Sep \\
\hline $33 \ldots \ldots \ldots \ldots \ldots \ldots \ldots \ldots \ldots \ldots \ldots \ldots \ldots \ldots$ & D10 & 106DiH & -0.693 & -0.605 & 269 & 15 & 211 & 43 & 2004 Sep \\
\hline $34 \ldots \ldots \ldots \ldots \ldots \ldots \ldots \ldots \ldots \ldots \ldots \ldots \ldots \ldots$ & F22 & $107 \mathrm{ExH}$ & -1.397 & -1.643 & 268 & 5 & 221 & 42 & 2004 Sep \\
\hline $35 \ldots \ldots \ldots \ldots \ldots \ldots \ldots \ldots \ldots \ldots \ldots \ldots \ldots \ldots \ldots$ & F23 & $108 \mathrm{ExH}$ & v0.536 & -1.891 & 259 & 13 & 185 & 61 & 2004 Sep \\
\hline $36 \ldots \ldots \ldots \ldots \ldots$ & $\mathrm{F} 24$ & 109ExH & -0.531 & -1.470 & 251 & 27 & 174 & 50 & 2004 Sep \\
\hline 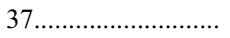 & F17 & $110 \mathrm{HaS}$ & -2.177 & -2.402 & 157 & 27 & 10 & 120 & 2004 Sep \\
\hline $38 \ldots \ldots \ldots \ldots \ldots \ldots \ldots \ldots \ldots \ldots \ldots \ldots \ldots$ & F18 & $111 \mathrm{HaS}$ & -2.108 & -2.198 & 123 & 15 & 10 & 98 & 2004 Sep \\
\hline 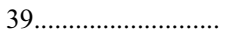 & F25 & $123 \mathrm{GlS}$ & 3.080 & -3.161 & 104 & 3 & 20 & 81 & 2005 Oct \\
\hline 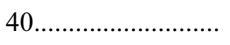 & F26 & 124G1S & 3.048 & -3.127 & 109 & 4 & 21 & 84 & 2005 Sep \\
\hline $41 \ldots \ldots \ldots \ldots \ldots \ldots \ldots \ldots \ldots$ & D12 & 131DiH & 0.653 & 0.852 & 289 & 3 & 39 & 227 & 2005 Oct \\
\hline $42 \ldots \ldots \ldots \ldots \ldots \ldots \ldots \ldots$ & D14 & 134DiH & 0.867 & 1.115 & 279 & 27 & 25 & 227 & 2005 Oct \\
\hline $43 \ldots \ldots \ldots \ldots \ldots \ldots \ldots \ldots$ & D15 & 135DiH & 1.189 & 1.300 & 213 & 74 & 15 & 124 & 2005 Oct \\
\hline $44 \ldots \ldots \ldots \ldots \ldots \ldots \ldots \ldots$ & $\mathrm{F} 27$ & 137DiH & 1.338 & 1.581 & 262 & 99 & 21 & 142 & 2005 Oct \\
\hline $45 \ldots \ldots \ldots \ldots \ldots \ldots \ldots \ldots$ & F28 & 138DiH & 1.356 & 1.656 & 265 & 20 & 26 & 119 & 2005 Oct \\
\hline $46 \ldots \ldots \ldots \ldots \ldots \ldots \ldots$ & F21 & $148 \mathrm{ExH}$ & -1.080 & -2.045 & 213 & 17 & 116 & 80 & 2005 Sep \\
\hline 47 ........................... & F29 & $150 \mathrm{ExH}$ & 1.206 & 2.205 & 229 & 74 & 38 & 117 & 2005 Sep \\
\hline $48 \ldots \ldots \ldots \ldots \ldots \ldots \ldots \ldots$ & F30 & $151 \mathrm{ExH}$ & 1.695 & 2.396 & 195 & 43 & 16 & 136 & 2005 Sep \\
\hline 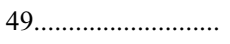 & F31 & $152 \mathrm{ExH}$ & 1.679 & 2.536 & 209 & 33 & 18 & 158 & 2005 Sep \\
\hline $50 \ldots \ldots \ldots \ldots \ldots \ldots \ldots \ldots$ & $\mathrm{S} 28$ & $153 \mathrm{ExH}$ & 0.1916 & -0.863 & 170 & 21 & 84 & 65 & 2005 Sep \\
\hline 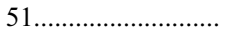 & D13 & 165DiH & 0.747 & 0.948 & 265 & 27 & 16 & 222 & 2005 Oct \\
\hline $52 \ldots \ldots \ldots \ldots \ldots \ldots \ldots \ldots \ldots \ldots \ldots \ldots$ & D11 & 166DiH & -0.6643 & -0.553 & 240 & 20 & 132 & 88 & 2005 Oct \\
\hline $53 \ldots \ldots \ldots \ldots \ldots \ldots \ldots \ldots$ & F20 & $167 \mathrm{HaH}$ & -1.597 & -1.838 & 209 & 16 & 128 & 65 & 2005 Oct \\
\hline $54 \ldots \ldots \ldots \ldots \ldots \ldots$ & F19 & $168 \mathrm{HaH}$ & -1.605 & -2.180 & 170 & 19 & 35 & 116 & 2005 Oct \\
\hline Total ...................... & & & & & 9861 & 1207 & 4032 & 4622 & \\
\hline
\end{tabular}

a Total number of stars with cross-correlation peaks greater than 0.05 .

b Halo stars: all stars lagging the peak of the disk velocity by $v_{\text {lag }}>160 \mathrm{~km} \mathrm{~s}^{-1}$, or when no disk is detected in outer halo fields, then from the average disk lag, $25 \mathrm{~km} \mathrm{~s}^{-1}$. From this sample of 1207 radial velocities, the giant stream is windowed out, and only stars with velocity errors $<20 \mathrm{~km} \mathrm{~s}{ }^{-1}$ are used, giving a final halo sample of 827 stars.

${ }^{\mathrm{c}}$ Disk stars (encompassing both thin and thick components, as described in the text): stars rotating with our disk model with a velocity lag $v_{\text {lag }}<160 \mathrm{~km} \mathrm{~s}^{-1}$.

d Stars with radial velocities falling within the "Galactic window," defined as $v_{\text {hel }}>-160 \mathrm{~km} \mathrm{~s}^{-1}$. Note that this column is only for consistent bookkeeping of the halo sample across fields, but many stars from this window are used for disk analyses (see, e.g., I05, and Fig. 2). The actual Galactic fraction of the total sample is estimated at $\sim 25 \%$. 

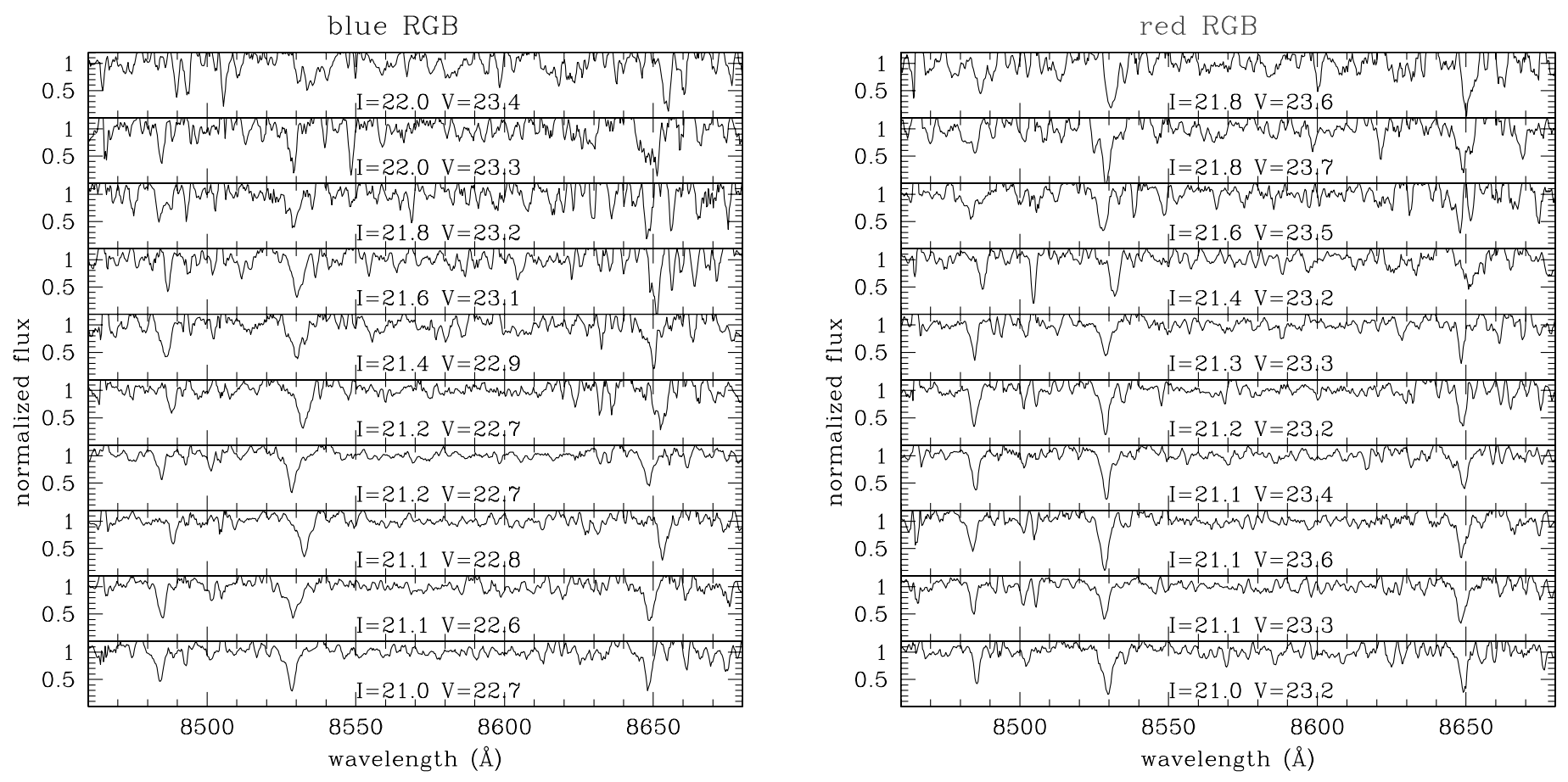

FIG. 3. - Representative spectra chosen from one of our survey fields (F3-w72), showing blue RGB stars with $V-I=1.3-1.8$ (left) and red RGB stars with $V-I=1.8-2.5$ (right), over the range of magnitudes for which we obtain good cross-correlations with the CaT template $(I<22)$. In this field there are no RGB candidates brighter than $I=21$, nor any good cross-correlations for stars fainter than $I=22$. [See the electronic edition of the Journal for a color version of this figure.]

adjusted accordingly by a small amount (hence the limits of the "disk" and "thick disk" selections in Fig. 2 are slightly ragged). The disklike population is revealed in Figure 4 as a Gaussian with disk-lag velocity of zero. Some skew in the distribution arises from the truncation in the northeast region of M31 imposed in order to reject contaminating stars from the Milky Way. This also affects the $v_{\text {hel }}$ visualization of the disk in Figure 4, giving rise to a much larger $-470 \mathrm{~km} \mathrm{~s}^{-1}$ disk peak (whereas the disk is actually sampled with roughly even numbers from our spectroscopy).

The minor axis fields require an additional windowing criterion. For inner fields where the rotating components dominate, we have windowed out the "disk" component as it passes over $v_{\text {hel }}=-300 \mathrm{~km} \mathrm{~s}^{-1}$ (even though this removes any possible halo sample as well - clearly this is unavoidable). For outer fields where the halo dominates, we do not window at all. The first such field is F5 at $20 \mathrm{kpc}$ on the southeast minor axis (Fig. 1), and all fields beyond that were considered to be "pure halo."

We do not explicitly distinguish a bulge component in this study, as it does not obviously distinguish itself in our predominantly major axis fields. We note that a relatively metal-rich bulgelike component is reported at minor axis radii from $12-20 \mathrm{kpc}$ by Kalirai et al. (2006b) consistent with other photometric studies of RGB stars at these radii (Durrell et al. 1994, 2001; Mould \& Kristian 1986; Brown et al. 2003; Bellazzini et al. 2003; Worthey et al. 2005). However, this is not crucial to the present study. Very few of our fields lie within this radial range, and even fewer are used for measuring the halo spectroscopic metallicity (see $\S 4$ and Fig. 9, which shows the fields where the numbers and quality of the spectra are sufficient for obtaining a halo metallicity measurement). As discussed above, our first minor axis field suitable for direct comparison with the Kalirai et al. (2006b) bulge is F5 at $20 \mathrm{kpc}$. This field has a halo sample metallicity of $[\mathrm{Fe} / \mathrm{H}]=-1.18$ (to be presented in Fig. 9), and is consistent with the findings of Kalirai et al. (2006b) within errors of their bridging halo/bulge fields. To ensure that this result is not biased by Galactic dwarf star contamination, we have tested the field F5 with velocity cuts at both -300 and $-170 \mathrm{~km} \mathrm{~s}^{-1}$, The resulting measurements are $[\mathrm{Fe} / \mathrm{H}]=-1.23(v<-300 \mathrm{~km} \mathrm{~s})$ and $[\mathrm{Fe} / \mathrm{H}]=-1.18(v<-170 \mathrm{~km} \mathrm{~s})$. This is in the opposite sense from that expected if dwarf contaminants were significant (see below). Our current inability to clearly distinguish a possible bulge component in the innermost regions of M31 may bias our measurements to slightly higher metallicity, although there is no statistically significant evidence that this is the case.

The Galactic foreground (discussed below) and the giant southern stream are the last contaminants to window from our stellar halo sample. The giant southern stream has kinematics that stand out as a high negative velocity peak in Figure 2 (Ibata et al. 2004). It appears in only seven of the fields, although another eight fields in the northeast region show a coherent kinematic structure that is similarly metal-rich to the giant southern stream, suggesting that this region represents a dominant component of the stream's return on the other side of M31 (Chapman et al. 2006). Nonetheless, the total number of stars in the giant stream is still relatively small, leaving the extended rotating component windows having the most significant effect on the halo sample.

The sample selection in the disk-lag and heliocentric frames is shown in Figure 2 (where the black dots mark the halo stars of interest to this study) and Figure 4 where the total distributions are shown. Figure 4 reveals the stellar halo windowed sample as a broad Gaussian distribution centered on $v_{\text {hel }}-300 \mathrm{~km} \mathrm{~s}^{-1}$, along with the rotating thin- and thick-disk-like populations together as a single sample. Also shown in Figure 4 are representative velocity histograms in nine individual fields spread throughout M31. The double-peaked disk/Galaxy components seen in the top three panels (from the northeast region of M31F30, F10, F8) demonstrate that our $v_{\text {hel }}<-160 \mathrm{~km} \mathrm{~s}^{-1}$ Galactic windowing is indeed very conservative, yet necessary to obtain an uncontaminated stellar halo sample. 

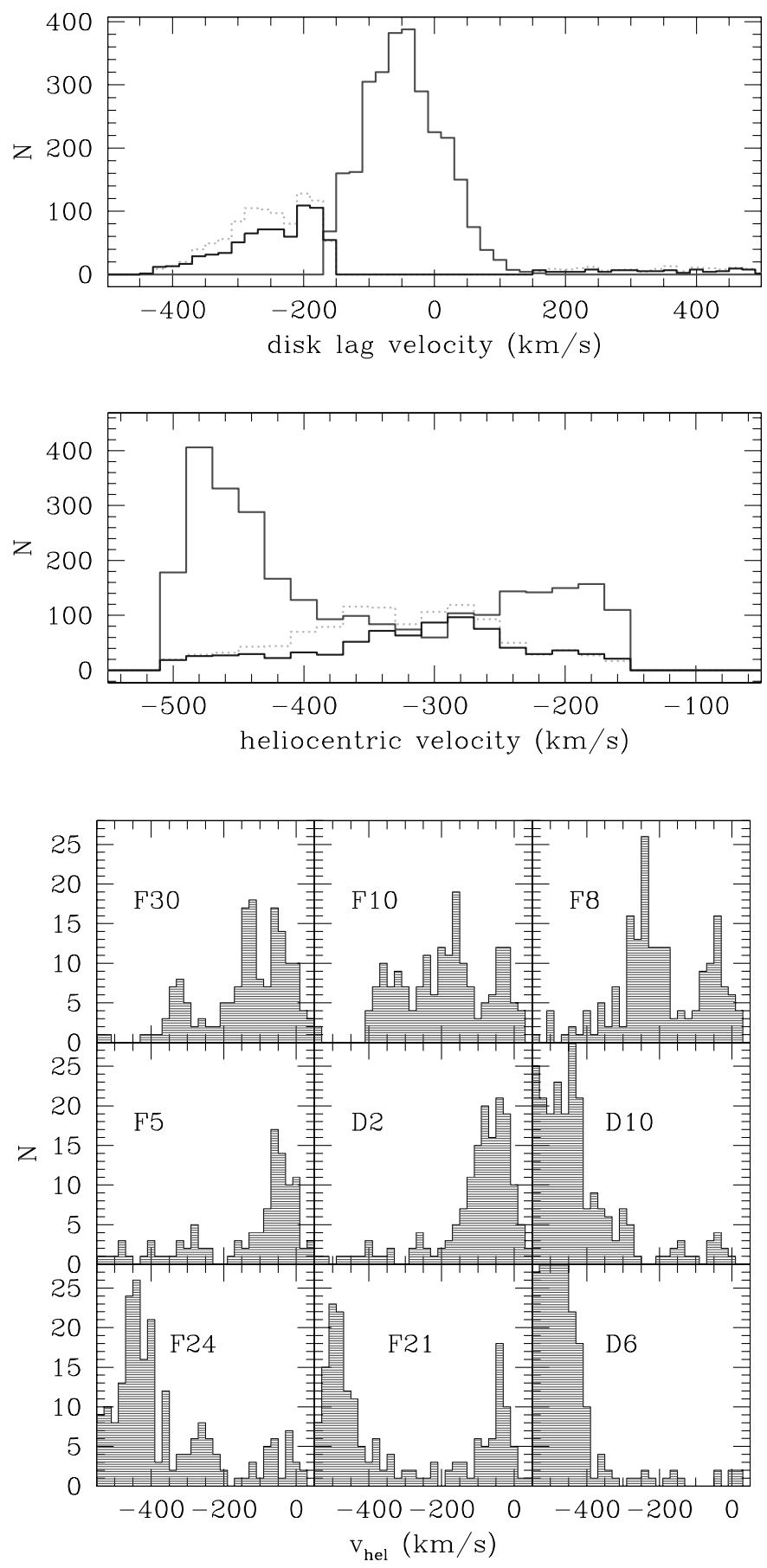

FIG. 4.-Top: Histograms of the halo/disk populations shown in Fig. 2, where thin- and thick-disk populations have been combined into a single histogram. Top: The kinematic sample selection, defined in the frame of disk-lag velocities, with the disk-like population (shown in red) centered at zero. The halo-like population is chosen to be effectively nonrotating $\left(v_{\text {disklag }}>160 \mathrm{~km} \mathrm{~s}^{-1}\right)$. The halo sample is shown both with (dotted histogram) and without (solid black histogram) the metal-rich kinematically coherent structure in the northeast region, which is likely to be associated with the giant southern stream (Chapman et al. 2006). Middle: The same samples are shown in the heliocentric frame, highlighting the broad halo-like Gaussian distribution centered on the M31 systemic velocity. Bottom: Representative histograms of nine individual fields spread throughout M31 (labeled as in Fig. 1), and arranged roughly by location in M31. The doublepeaked disk/Galaxy components in the top three panels (from the northeast region of M31-F30, F10, F8) reveal that our $v_{\text {hel }}<-160 \mathrm{~km} \mathrm{~s}^{-1}$ Galactic windowing is indeed very conservative, but deemed necessary to procure a nearly pure halo sample. [See the electronic edition of the Journal for a color version of this figure.]
After all windowing procedures, 1207 stars remain in our halo sample. We then refine this sample to 827 stars by applying the following quality criteria (the same criteria as in I05): (1) The velocity of stars derived from two different sky-subtraction algorithms has to agree within $20 \mathrm{~km} \mathrm{~s}^{-1}$; this removes 35 stars. (2) Stars with continuum $>10$ counts (or about $40 e^{-}$total pixel ${ }^{-1}$ ); this removes 38 stars. (3) Stars with $\sigma_{v}<20 \mathrm{~km} \mathrm{~s}^{-1}$ : this removes 138 stars. (4) Stars with cross-correlation peak $<0.05$; this removes 189 stars. This sample can be considered to underlie the extended rotating structure of $\mathrm{I} 05$.

\subsection{Galactic Dwarf Contamination}

To complete our halo sampling, we must remove the foreground Galactic component (mostly dwarf stars), observed as a strong Gaussian peak centered on $-61 \mathrm{~km} \mathrm{~s}^{-1}$ (I05). The Galactic window is stationary (defined here as stars with $v_{\text {hel }}>$ $-160 \mathrm{~km} \mathrm{~s}^{-1}$ ), except for fields within the dense disk of M31 on the northeastern major axis where we chose to remove only those stars with $v_{\text {hel }}>0 \mathrm{~km} \mathrm{~s}^{-1}$ as the source density is so high that Galactic contaminants are rare. Any Galactic contamination near the M31 systemic velocity $\left(v_{\text {hel }}=-300 \mathrm{~km} \mathrm{~s}^{-1}\right)$ has to be Galactic halo, and the corresponding density of dwarf stars is minimal. The Besançon Galactic starcounts model (Robin et al. 2004) estimates 10 Galactic stars per M31 DEIMOS field of view $\left(16.7 \times 5^{\prime}\right)$ with $v_{\text {hel }}<-160 \mathrm{~km} \mathrm{~s}^{-1}$ if all available candidates were observed. For $v_{\text {hel }}<-300 \mathrm{~km} \mathrm{~s}^{-1}$ the Besançon model indicates the number of Galactic stars per DEIMOS field to be only 1.8 stars (typically $\ll 1 \%$ ). The Besançon prediction at velocities $<-300 \mathrm{~km} \mathrm{~s}^{-1}$ is particularly relevant since more than $50 \%$ of our halo sample is cut at $v_{\text {hel }}<-300 \mathrm{~km} \mathrm{~s}^{-1}$. In practice, we have typically observed $<50 \%$ of the stars that are consistent with M31 RGB colors available in the region covered by a DEIMOS mask. Given our choice of thousands of targets per field inside the inner ellipse in Figure 1 (and a maximum multiplexing of $\sim 600$ stars per DEIMOS mask with our megaholes approach; see I05 for further discussion), we estimate a negligible Galactic contamination out to $\sim 30 \mathrm{kpc}$, and at most $10 \%$ contamination in our fields at larger radius. We also refer to the work of Gilbert et al. (2006), which uses a multidimensional rejection of Galactic dwarfs based on a variety of spectral features (most of which are unavailable to us, given the small extracted wavelength regime from our custom data reduction). Gilbert et al. (2006) are therefore able to calibrate the use of kinematic selection alone as a means to reject Galactic dwarfs, finding that $<1 \%$ of stars cut at $v_{\text {hel }}<-300 \mathrm{~km} \mathrm{~s}^{-1}$ will be dwarfs, in agreement with the Besançon model. Therefore our CMD selection of RGB stars coupled with kinematic sorting of the candidate M31 stars by radial velocity is likely to remove the vast majority of Galactic contaminants.

The windowing of stellar halo sample provides another check on Galactic contamination. Our northeastern stellar halo represents a more "Galactic dwarf-free" sample as it is cut in velocity below $-300 \mathrm{~km} \mathrm{~s}^{-1}$, whereas the southwestern stellar halo is cut typically from $-160 \mathrm{~km} \mathrm{~s}<v_{\text {hel }}<-300 \mathrm{~km} \mathrm{~s}^{-1}$. As we might expect based on the Gilbert et al. (2006) work, we find almost no differences between our northeast and southwest hemisphere results, neither in kinematics ( $(3)$ nor in metallicity $(\S 4)$, and thus it is clear that Galactic dwarfs are not significantly affecting our stellar halo results.

A further reality check that our spectroscopically identified stars are truly M31 RGB stars comes from the $\mathrm{Na}$ I $\lambda \lambda 8183$, 8195 , which is sensitive to surface gravity and is accordingly undetectable in M31 RGB stars, but is as strong as the CaT absorption lines in Galactic dwarfs with relatively red colors 

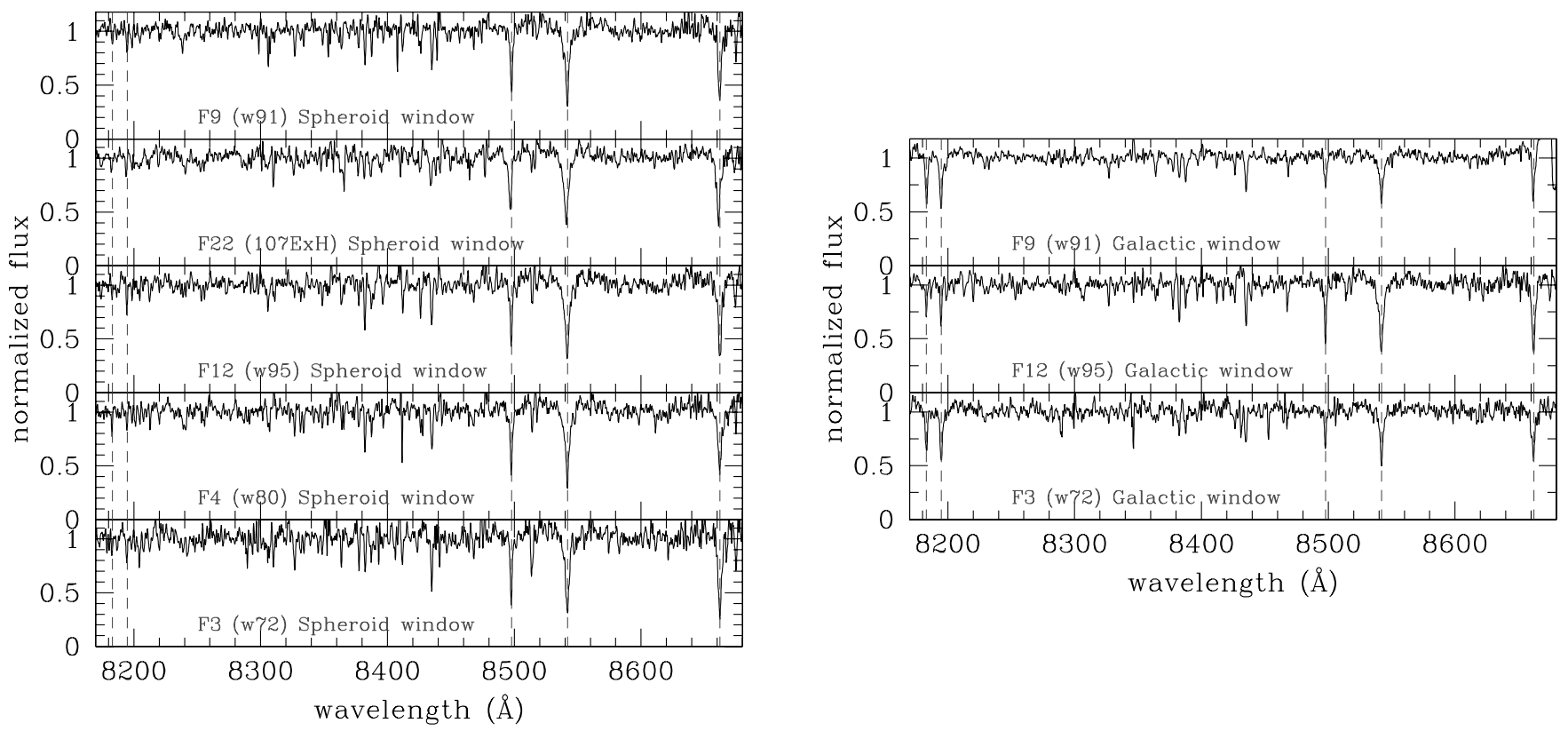

FIG. 5.- Averaged spectra with wavelength coverage below $8200 \AA$ for typical DEIMOS fields. The left panels show halo samples from both northeast and southwest regions of M3, and therefore having velocity windows ranging from $-160 \mathrm{~km} \mathrm{~s}^{-1}>v_{\text {hel }}>-300 \mathrm{~km} \mathrm{~s}^{-1}(\mathrm{~F} 3, \mathrm{~F} 4)$ to $-300 \mathrm{~km} \mathrm{~s}^{-1}>v_{\text {hel }}>-500 \mathrm{~km} \mathrm{~s}{ }^{-1}(\mathrm{~F} 12, \mathrm{~F} 9, \mathrm{~F} 22)$. These fields show strong CaT absorption and minimal Na I $\lambda \lambda 8183,8195$ absorption. Similar results are found in all halo samples, providing a reality check on the minimal Galactic contamination to these samples at high negative velocities. The right panel shows the average of stars drawn from the $v_{\text {hel }}<-100 \mathrm{~km} \mathrm{~s}^{-1}$ region thought to be highly contaminated by Galactic dwarf stars in three of the same fields. These stars show a strong Na I absorption typical of Galactic dwarfs with redder colors (e.g., Guhathakurta et al. 2006), with weaker CaT absorption than seen in the metal-poor halo samples. There are between 10 and 16 stars in each stack. [See the electronic edition of the Journal for a color version of this figure.]

(e.g., Guhathakurta et al. 2006). We can check for this on a field-by-field basis in combined spectra of stars, and where our spectroscopic approach resulted in wavelength coverage to $<8200 \AA$ (the $\sim 30 \%$ of fields where we employed the standard DEIMOS multislit masks). Our stacked spectra in all M31 components (extended disk, thick disk, halo, and giant stream) show very little evidence for $\mathrm{Na}$ I 28183,8195 absorption lines, whereas stars drawn randomly from -100 to zero velocity show strong $\mathrm{Na}$ I absorption on average (Fig. 5). Direct measurement of the $\mathrm{Na}$ I equivalent widths in the halo and Galactic windows shows an upper limit on the contamination fraction of $15 \%$, although this is of course a severe upper limit since there are large numbers of true M31 RGBs in the "Galactic" window (Gilbert et al. 2006) and weak $\mathrm{Na}$ I is expected in RGBs. This further corroborates the very low Galactic contamination in our M31 kinematically selected samples.

\section{RESULTS: KINEMATIC PROPERTIES OF THE STELLAR HALO}

To provide an overall kinematic characterization of the M31 halo stars, we first fitted a simple model with an isotropic velocity dispersion that is independent of radius, but that is allowed to rotate as a solid body about the center of the galaxy, which is assumed to have a systemic heliocentric velocity of $-300 \mathrm{~km} \mathrm{~s}^{-1}$, and with the same axis of rotation as the disk. For this first fit, we manually removed three fields from the sample, due to the obvious presence of a kinematic halo substructure. These three contiguous fields lie on the northeastern major axis at the edge of the inner ellipse shown in Figure 1, and the kinematic substructure is clearly visible on Figures 2 and 4 with heliocentric velocity in the range $-400<v_{h}<-350 \mathrm{~km} \mathrm{~s}^{-1}$ (this detection is is discussed further in Chapman et al. 2006). The model was fit simultaneously to the halo stars in all of the remaining 51 spectroscopic field using a windowed maximum likelihood approach. The resulting distribution of likelihood as a function of the model dispersion and angular speed is shown in Figure 6. The best model parameters given this data are a halo velocity dispersion of $\sigma_{v}=126 \mathrm{~km} \mathrm{~s}^{-1}$, and an angular velocity of $\Omega=$ $-0.08 \mathrm{~km} \mathrm{~s}^{-1} \mathrm{kpc}^{-1}$. However, the data are also consistent, at the $1 \sigma$ confidence level, with no rotation.

Indeed, the halo shows no significant signs of rotation in any orientation. By dividing the spectroscopic fields into two subsamples using a line intersecting the center of M31, we find the largest velocity differential as a function of azimuthal angle occurs near the major axis where the northeast half shows $v_{\text {hel }}=$ $-292 \pm 8 \mathrm{~km} \mathrm{~s}^{-1}$ from a best-fit Gaussian to the average sample,

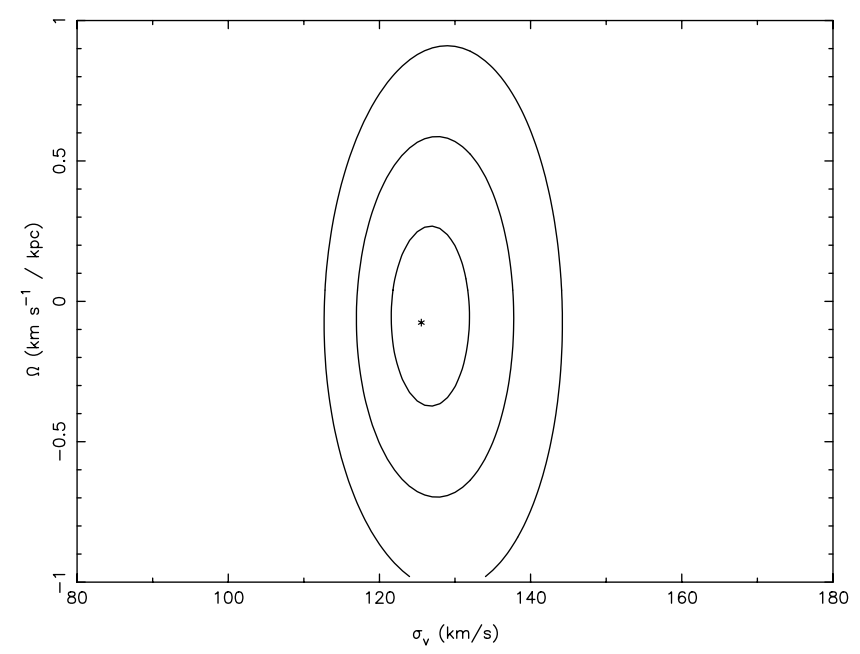

FIG. 6.- Likelihood contours (at 1,2, and $3 \sigma$ intervals) of a simple rotating isothermal halo model, as a function of velocity dispersion and rotation. The asterisk denotes the position of the most likely parameters $\sigma_{v}=126 \mathrm{~km} \mathrm{~s}^{-1}$, $\Omega=-0.08 \mathrm{~km} \mathrm{~s}^{-1} \mathrm{kpc}^{-1}$, where positive $\Omega$ denotes motion in the same sense as the galactic disk. The data are clearly consistent with zero halo rotation at the $1 \sigma$ level. 

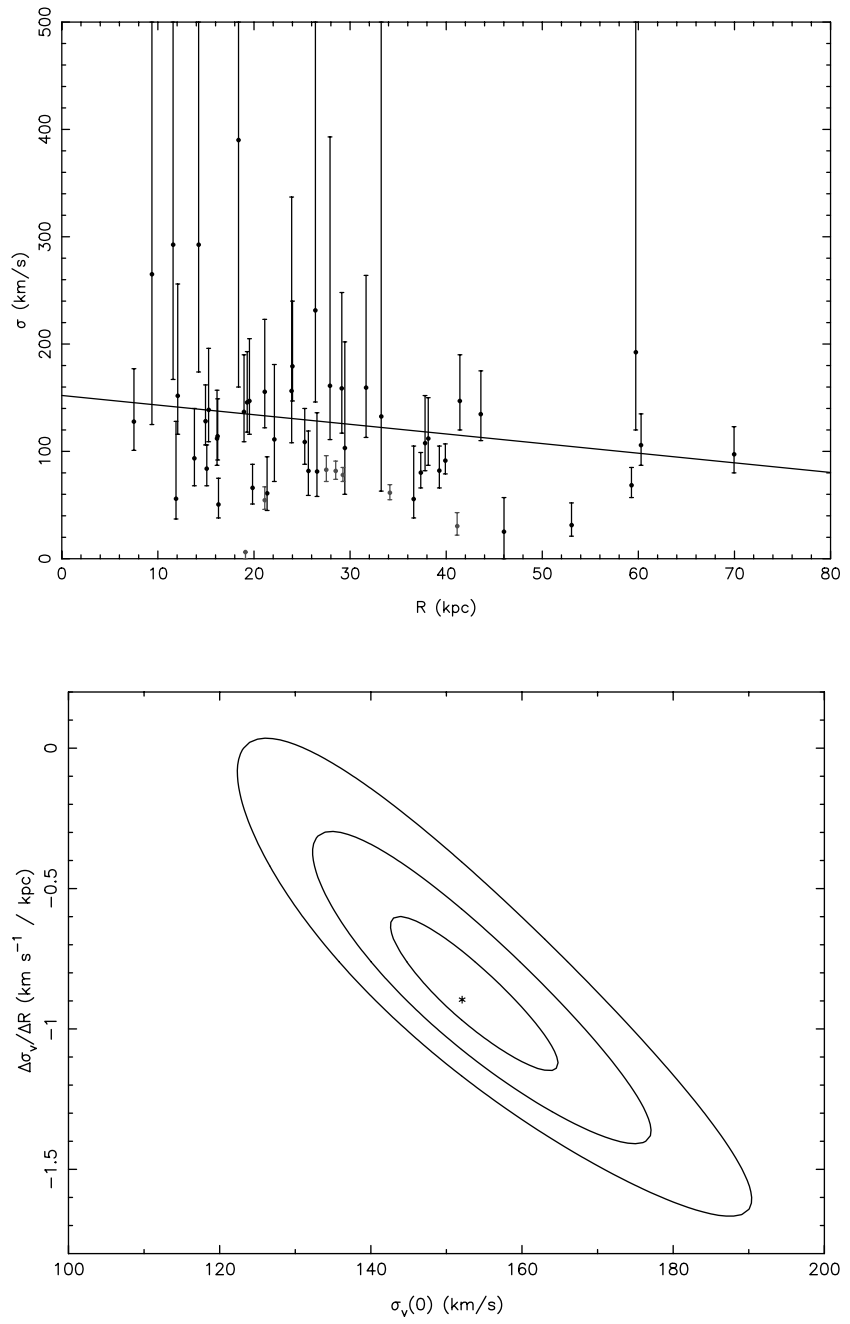

FIG. 7.-Top: Explicit single dispersion fits to the halo stars in the DEIMOS fields as a function of projected radius $R$. The vertical bars show $1 \sigma$ uncertainties in these maximum-likelihood fits. The substantial variations in the fitted values and their uncertainties are to a large extent due to the low number of halo stars in most fields, and due to the different window functions in each field. Bottom: Likelihood contours (at 1,2, and $3 \sigma$ intervals) for a nonrotating halo model in which the velocity dispersion is allowed to decrease linearly with projected radius $R$. The most likely central velocity dispersion of this model is $\sigma_{v}=152 \mathrm{~km} \mathrm{~s}^{-1}$, which decreases with radius $R$ as $-0.90 \mathrm{~km} \mathrm{~s}^{-1} \mathrm{kpc}^{-1}$ (the straight line corresponding to these values is displayed in the top panel). The fields in the top panel that have a likelihood of less than $1 \%$ of being consistent with this line were rejected in an iterative manner to produce this fit. [See the electronic edition of the Journal for a color version of this figure.]

while the southwest half has $v_{\text {hel }}=-303 \pm 6 \mathrm{~km} \mathrm{~s}^{-1}$. This insignificant rotation is in the same sense as the extended rotating component (Ibata et al. 2005), and could for instance be a result of residual extended rotating component stars contaminating our halo sample. For simplicity we therefore neglect rotation in the following, slightly more complex models.

In Figure 7 we show the result of fitting a model in which the velocity dispersion is allowed to vary linearly with projected radius $R$. The systemic velocity was again fixed at $-300 \mathrm{~km} \mathrm{~s}^{-1}$ for stability due to a complex windowing function. We proceeded by first calculating the velocity dispersions in individual fields, given the adopted window function, and assuming that the dispersion is constant and Gaussian at the position of the field. The resulting maximum-likelihood calculated values are shown in the top panel of Figure 7. A single model with a linear dependence of velocity dispersion with radius was then fit to the full data set. The measurements for individual fields are not used in the analysis, presented only to clarify what the maximumlikelihood algorithm is latching onto. Proceeding in an iterative manner, we rejected those fields where the likelihood of the velocity dispersion conforming to the linear fit was less than $1 \%$, and recomputed the fit. The likelihood contours of this model are shown in the bottom panel of Figure 7; the most likely model has

$$
\sigma_{v}(R)=152-0.90 \frac{R}{1 \mathrm{kpc}} \mathrm{km} \mathrm{s}^{-1} \mathrm{kpc}^{-1} .
$$

At a distance of $10 \mathrm{kpc}$, the inferred velocity dispersion of the halo is therefore $\sim 143 \mathrm{~km} \mathrm{~s}^{-1}$, while at a distance of $60 \mathrm{kpc}$ the inferred velocity dispersion has changed to $\sim 98 \mathrm{~km} \mathrm{~s}^{-1}$. These results stand in contrast to the essentially isothermal halo measured for the inner Galaxy (Chiba \& Beers 2000); for M31, an isothermal model can be rejected at the $\sim 3 \sigma$ level.

The individual field-by-field fits reveal that the overall fit when we allow for a gradient in the dispersion is obviously dominated by the stars inside $40 \mathrm{kpc}$. While stars likely belonging to the giant stream have been removed from the halo sample, several stream fields stand out very clearly as not belonging to the trend by having a very low velocity dispersion, as do fields in the northeast shelf region (described in Ferguson et al. [2002], which also shows stars with giant stream kinematic structure). The fact that these fields still stand out from an otherwise well-behaved trend suggests that our windowing of the giant stream is not extensive enough. However, at the present time we lack a full understanding of the distribution of the stream debris over the face of M31, forcing us to adopt this pragmatic approach.

The above two models are essentially just phenomenological descriptions of the data, so in the following we attempt a more physically motivated model. Modern cosmology finds that the dark matter halos of galaxies have a universal shape (Navarro et al. 1997 hereafter NFW). Simulations show that halo stars are the remnants of ancient accreted protogalaxies, but ones that were relatively massive. As such the stars deposited by these accretions are biased toward being closer to the center of the halo, and consequently the halo is less extensive than the dark matter halo (Abadi et al. 2006). Nevertheless, the halo stars provide a reasonable first approximation to the dark matter, and we will therefore attempt to fit the population with an NFW model. This comparison is further motivated by the finding that the velocity dispersion of the halo sample decreases with radius, which is expected from the NFW model.

The NFW model is fully described by concentration and virial mass parameters. Although the concentration is on average dependent on the virial mass (Bullock et al. 2001), there is a large scatter in this relation, and it is therefore safer to consider the full parameter space. We integrated the NFW model numerically, using the relations described in Lokas \& Mamon (2001) to obtain the projected line of sight velocity dispersion for each field in our survey, under the assumption that the velocity dispersion is isotropic. Note that the relative densities between fields were purposefully not specified, due to the fact our survey does not provide a complete census of stars in each field. We further adopted $H_{0}=70 \mathrm{~km} \mathrm{~s}^{-1}$ and a virial overdensity parameter of 200 . Figure 8 shows the resulting likelihood contours (after rejection of the low-dispersion fields marked red in the top panel of Fig. 7). Although the most likely model parameters are $M_{V}=$ $9.8 \times 10^{11} M_{\odot}$ and $c=38$, the likelihood contours are very extended in the concentration direction. The main reason for this is that our maximum-likelihood analysis method, as mentioned before, does not have access to the information on the field-to-field 


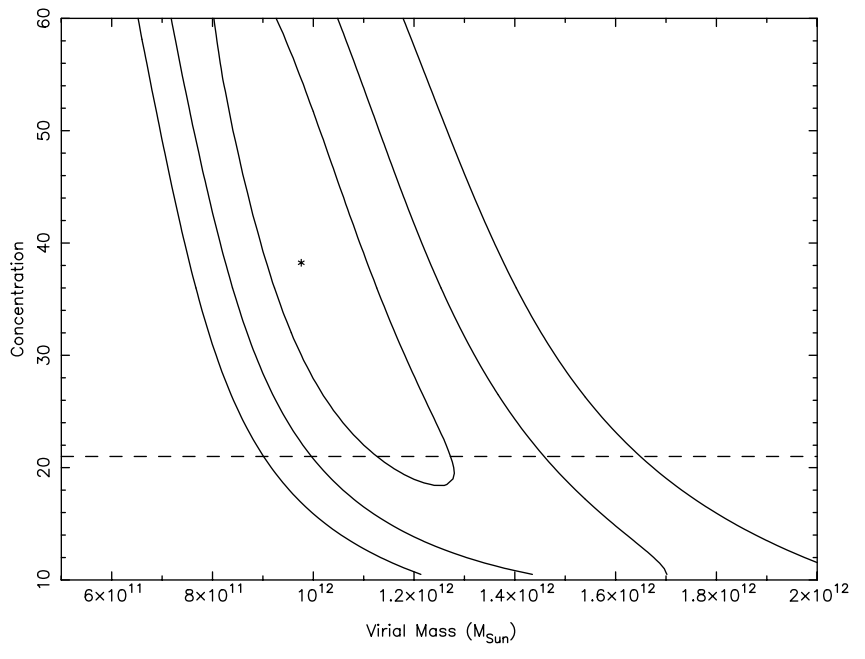

FIG. 8.- Likelihood contours (at 1, 2, and $3 \sigma$ intervals) of an NFW model fit to our halo star sample, after rejection of the low-dispersion fields identified from the analysis presented in Fig. 6. Here the most likely model has a virial mass of $M_{V}=9.8 \times 10^{11} M_{\odot}$ and concentration $c=38$. Notice however, that the constraint on the model concentration provided by our analysis is not strong, and substantially lower concentration values are allowed (lower than $c=18$ within the $1 \sigma$ boundary). The dashed line shows the $c=21$ upper limit to the concentration of an M31-like galaxy argued by Klypin et al. (2002). Using this additional constraint, the virial mass of the M31 halo must be greater than $M_{V}=9.0 \times 10^{11} M_{\odot}$ with $99 \%$ confidence.

density variations, whereas the concentration by its very nature relates to the radial variation in density. What density sensitivity we do have is due to the integration along the line of sight in each field. Cosmological simulations indicate that concentration values of $c>21$ are very unlikely for the dark halos of galaxies such as M31 (Klypin et al. 2002); this limit is shown by a dashed line in Figure 8. Imposing this additional constraint implies that the virial mass of the M31 halo must exceed $M_{V}=9.0 \times 10^{11} M_{\odot}$ with $99 \%$ confidence. This value for the halo mass agrees remarkably well with the value calculated from the kinematics of the giant stream around M31 (Ibata et al. 2004), where we found a mass interior to $125 \mathrm{kpc}$ (the apocenter of the stream) of $M(R<125 \mathrm{kpc})=7.5_{-1.3}^{+2.5} \times 10^{11} M_{\odot}$. In contrast, the present constraint translates to $M(R<125 \mathrm{kpc})>7.2 \times 10^{11} M_{\odot}$.

\section{RESULTS: METALLICITY PROPERTIES OF THE STELLAR HALO}

The photometrically dominant extended rotating component appeared to have a relatively high metallicity, $[\mathrm{Fe} / \mathrm{H}]=-0.9$, $\sigma=0.2 \operatorname{dex}$ (I05), consistent with our larger sample of disk stars reported here, $[\mathrm{Fe} / \mathrm{H}]=-1.0 \sigma=0.3 \mathrm{dex}$ (where the error bars represent field to field dispersion in the measurements), explaining why so many disklike stars have been found at large radius (Reitzel \& Guhathakurta 2002; Reitzel et al. 2004). While attempts to model a more metal-rich halo have had some degree of success under specialized evolutionary conditions (Font et al. 2006), our CDM understanding of how the halo of a typical L* spiral galaxy forms suggests there must be a metal-poor relic of the early halo.

But is there perhaps a metal-poor halo lurking underneath the relatively metal-rich rotating component? Our first goal is to assess the metallicity of our velocity-windowed sample of stars, to see if it shows properties distinct from the prominent extended rotating component, which vastly outnumbers the halo (on average by a factor of $\sim 6$ ) at radii $<50 \mathrm{kpc}$ (Irwin et al. 2005).

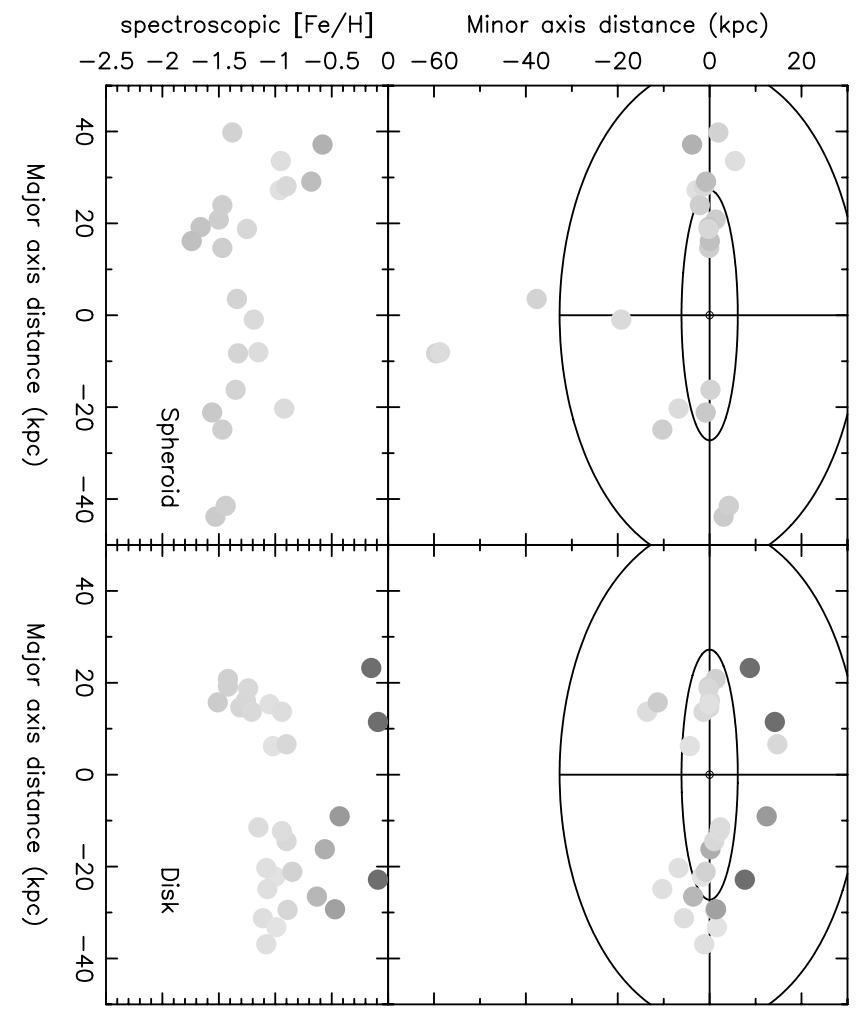

FIG. 9.- Metallicity measurements from stacked spectra are shown for the halo sample (left panels) and disk sample (right panels). The top panels show the spatial location of the fields, with the ellipses denoting the same limits as in Fig. 1. The smaller number of fields compared to Fig. 1 is due to the requirement that at least 10 well-measured spectra of the same galactic component be present in a field to provide a metallicity measurement. The five metal-rich halo fields in the northeast are not typical for the halo sample, and are clearly the result of contamination from a $[\mathrm{Fe} / \mathrm{H}] \sim-0.7$ dex kinematically coherent structure at $\sim-380 \mathrm{~km} \mathrm{~s}^{-1}$ which appears in all these fields, likely to be associated with the giant southern stream (Chapman et al. 2006). [See the electronic edition of the Journal for a color version of this figure.]

The DEIMOS spectra allow a measurement of the metallicity of the targeted stars from the equivalent widths (EWs) of the $\mathrm{Ca}$ II triplet absorption lines, free from the model dependency of photometric analysis. While the noise in individual spectra is typically too high to yield a useful measurement of the Ca II equivalent widths (whereby individual spectra can have vastly different systematic errors due to the inopportune location of sky lines for certain heliocentric velocities), we estimate the average metallicity by stacking the RGB star spectra in individual fields. We shift each spectrum to zero velocity before stacking, normalizing the continuum to one using the inter-CaT continuum regions to fit a spline function, and finally weighting individual spectra by the continuum S/N (Figs. 9 and 10). Metallicities were measured in an identical way to $\mathrm{I} 05$, using the correlation of the $\mathrm{Ca}$ II equivalent width with $[\mathrm{Fe} / \mathrm{H}]$ on the Carretta \& Gratton (1997) as calibrated by Rutledge et al. (1997).

For comparison, we derived photometric [ $\mathrm{Fe} / \mathrm{H}]$ values for the halo stars by interpolating between globular cluster fiducial sequences described in I05. The photometric properties of these metal-poor halo RGBs are entirely consistent with the spectroscopic properties, as shown in Figure 11 (we defer the model/ age-dependent analyses of the stellar photometry to a followup paper). The largest variations occur in the northeast fields where the metal-rich giant stream structure is present at the halo window velocities $\left(v_{\text {hel }}<-300 \mathrm{~km} \mathrm{~s}^{-1}\right)$, which appear to be more metalrich in the spectroscopic measurements. This may be indicative 


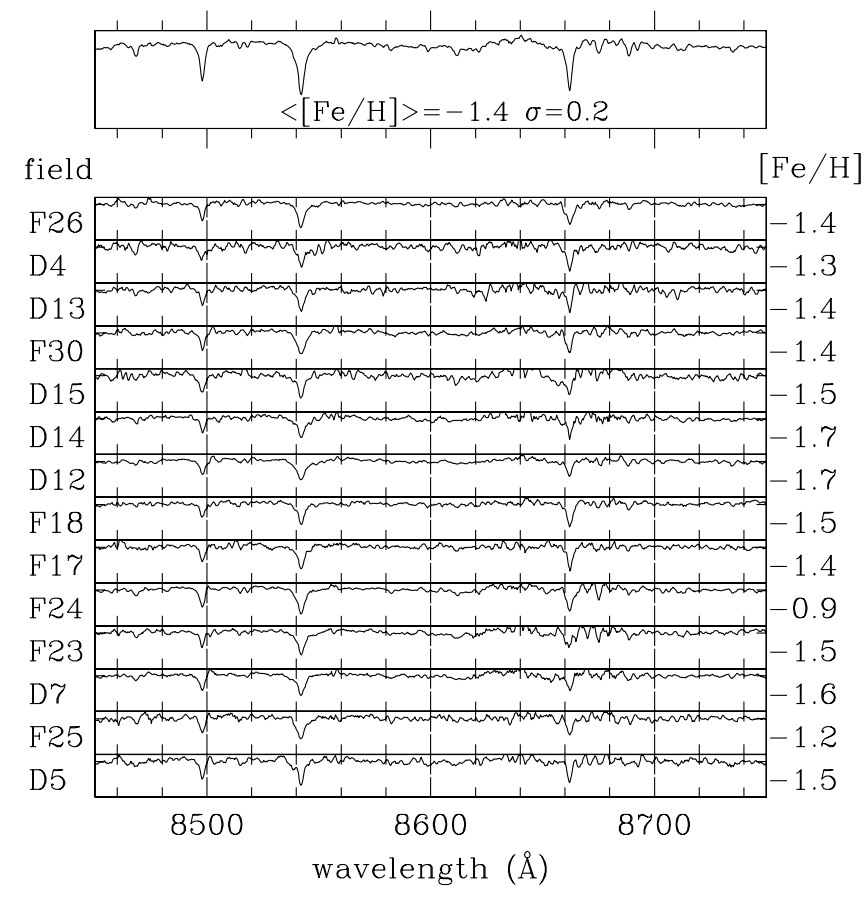

FIG. 10.- Representative combined spectra (weighted by the $\mathrm{S} / \mathrm{N}$ of individual spectra in the stack) for kinematically selected halo stars in 14 of the 23 fields shown in Fig. 9, along with the total stacked halo spectrum at the top exhibiting an average $[\mathrm{Fe} / \mathrm{H}]=-1.4 \sigma=0.2 \mathrm{dex}$, the error bar indicating the field to field rms variation (whereas measurement errors in the stacked spectra are much smaller). Field names and metallicities are indicated on the left and right, respectively. The spectra have been normalized to a continuum of one with the ordinate crossing at zero, and smoothed to the DEIMOS instrumental resolution. The three Ca II triplet lines at 8498.02, 8542.09, and 8662.14 $\AA$ are the most obvious features of these spectra, although many other real features are present.

of the different ages/properties of the giant stream stars from the stellar halo.

The average CaT triplet metallicity in stacked spectra with at least 10 high-quality halo stars, is $[\mathrm{Fe} / \mathrm{H}]=-1.4, \sigma=0.2$ (where the error quoted is simply the dispersion in the individual field measurements), much poorer than we find for the extended rotating component defined in the same manner $[\mathrm{Fe} / \mathrm{H}]=-1.0$, $\sigma=0.3$. There are five halo fields in the northeast that appear significantly more metal-rich than average and are not typical for the halo sample. These five fields all possess a kinematically coherent substructure at $v_{\text {hel }} \sim-380 \mathrm{~km} \mathrm{~s}^{-1}$ and with $[\mathrm{Fe} / \mathrm{H}] \sim$ -0.7 , which contaminates the halo-selected sample to various degrees. Including these substructure stars in the halo sample only marginally changes the result $([\mathrm{Fe} / \mathrm{H}]=-1.36, \sigma=0.32$ with, versus $[\mathrm{Fe} / \mathrm{H}]=-1.40, \sigma=0.21$ without). This substructure is likely to be associated with the giant southern stream (Ibata et al. 2001a, 2004).

Aside from these giant stream fields in the northeast, the metallicity measurements in each halo field are very similar, with no apparent radial gradient (Fig. 9). The three most outlying metallicities found are for fields D14 and D12 (both with halo $[\mathrm{Fe} / \mathrm{H}] \sim-1.7$ ) and for $\mathrm{F} 24$ (with halo $[\mathrm{Fe} / \mathrm{H}]=-0.9$ ), none of which can obviously be attributed to selection effects or contamination. Fields D14 and D12 are in the northeast hemisphere of M31, where as we have noted the halo sample cut with $v_{\text {hel }}<$ $-300 \mathrm{~km} \mathrm{~s}^{-1}$ results in $<1 \%$ Galactic contamination. While a small fraction of Galactic contamination is expected for F24 in the southeast hemisphere, this would in fact make the spectroscopic $[\mathrm{Fe} / \mathrm{H}]$ appear more poor, not rich. These fields may therefore host accreted substructures that are not as well phase

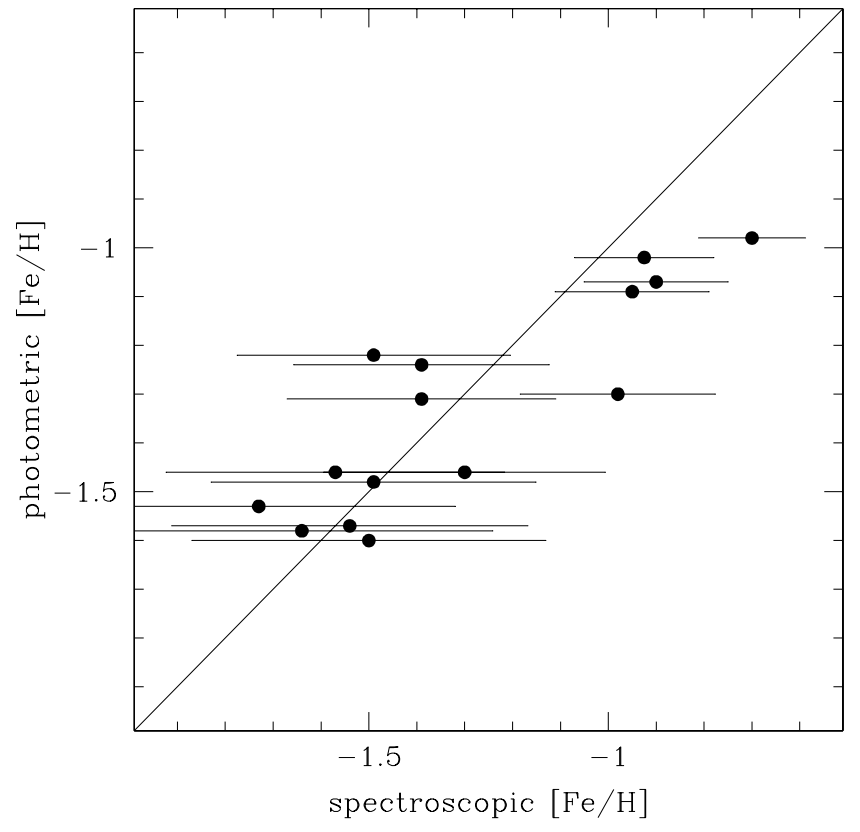

FIG. 11.-Comparison of the spectroscopic and photometric [ $\mathrm{Fe} / \mathrm{H}]$ derived for RGBs in the halo sample. Points are shown for all fields with at least 20 stars. The line denotes equality. Note that the photometric $[\mathrm{Fe} / \mathrm{H}]$ is highly age dependent; the good agreement with the spectroscopic $[\mathrm{Fe} / \mathrm{H}]$ suggests that the adopted $\sim 13$ Gyr ages adopted from the globular cluster fiducials is reasonable for the halo stars. The metal-rich points correspond to fields in the northeast region of the extended disk, where the giant southern stream (Ibata et al. 2001a) passes through at velocities within the halo window.

mixed as the halo in general. Our findings therefore suggest that there is a metal-poor, nonrotating halo component in M31, with comparable metallicity to the Milky Way's nonrotating stellar halo (Chiba \& Beers 2000, 2001).

The accuracy of our M31 halo metallicity measurement is limited by the continuum fitting in these relatively low signal-to-noise spectra, with additional uncertainty from systematic effects such as residual sky. The large distance of M31 implies a small uncertainty for the horizontal branch magnitude, even if the stars are drawn from relative extrema in the galaxy. Furthermore, the rms dispersion in $V$-band magnitudes of the total sample of halo stars is very small $(<0.5 \mathrm{mag})$. These effects diminish the impact on the derived metallicity of an uncertainty in distance in the RGB population we are observing in our sample. A formal error for each fit is obtained from the $\mathrm{S} / \mathrm{N}$ of the CaT lines, with an extra error estimated for the uncertainty in sky subtraction. The typical uncertainty is $\sim 0.3 \mathrm{dex}$.

A concern is that the average CaT metallicity in a given field may be dominated by a few outlying spectra, although this should only be a factor for increasing the metallicity (increasing the EW). This potential systematic error can be tested by dividing up the fields into halves and comparing metallicity measurements. Generally subsamples have metallicities consistent to within 0.2 dex. However, a danger with this approach is that for a small enough sample, variations will be present due to the random sampling of the underlying metallicity distribution.

Our sample of RGB stars isolated to have halo kinematics (and importantly windowing out the extended rotating component) have allowed a census of the halo along both major and minor axis fields, and underlying the range of photometric substructures observed. The uniformly low $([\mathrm{Fe} / \mathrm{H}]=-1.4, \sigma=0.2)$ metallicity we measure across the fields reflects a stellar halo component that has a distinctly different origin from the extended 
rotating component. Our discovery is complemented by the independent discovery of a halo component with metal poor $([\mathrm{Fe} / \mathrm{H}]=-1.5, \sigma=0.7)$ stars lying at large radius $(60-150 \mathrm{kpc})$ (Kalirai et al. 2006b; Gilbert et al. 2006). Their outer halo component and bridging halo/bulge component at smaller radii are similarly metal-poor to the halo we describe here. However, the 47 pure halo stars at $R>60 \mathrm{kpc}$ in Kalirai et al. (2006b) and Gilbert et al. (2006) lack sufficient statistics to ascertain whether they are consistent with an outer extension of the kinematically characterized, pressure-supported, nonrotating component we describe in this paper.

The larger field to field metallicity dispersion $(\sigma \sim 0.3)$ found by Kalirai et al. (2006b) \& Gilbert et al. (2006) is suggestive of an increasingly inhomogeneous halo component with radius, and is consistent with the dramatic structural variations from widefield photometric surveys of M31's outer halo by R. Ibata et al. (2006, in preparation) and Martin et al. (2006). These findings are also consistent with the much less phase-mixed outer halo in the simulations of Bullock \& Johnston (2005), suggesting that the outer halo may truly represent a component with a much longer assembly time than the inner halo.

\section{DISCUSSION}

While our study has uncovered conclusive evidence for a pressure supported metal-poor halo in M31 within the inner $70 \mathrm{kpc}$ (complemented by the codiscovery of a metal-poor halo in Kalirai et al. 2006b and Gilbert et al. 2006), evidence for metal-poor stars in Andromeda's halo reaches back to Holland et al. (1996), who used deep imaging in the outer halo to probe the halo CMD. Even earlier results from RR Lyrae studies suggested that old, metal-poor stars might be present in the halo (Pritchet \& van den Bergh 1988). Metal-poor RGB stars were first identified in the halo using Keck spectroscopy of by Reitzel \& Guhathakurta (2002); Reitzel et al. (2004) speculating that differences seemed to exist between subsets of the RGBs in their sample, finding a large range in metallicities, and suggesting that disklike stars were found at radial distances as large as $34 \mathrm{kpc}$. However, with only two isolated pointings and small numbers of stars (23 and 29, respectively), these studies had no means to characterize the outer structural components in M31.

While our Keck spectroscopic data do not suffer from age/ metallicity degeneracy as do the earliest photometric studies of the halo, the most important ingredient for our discovery is the detailed knowledge of the extended rotating component, which allows for the separation of populations by their kinematics. Thus when stars are selected in a similar way to the halo star selection in Galactic studies (i.e., by extreme kinematics; Chiba \& Beers 2000), a similar $[\mathrm{Fe} / \mathrm{H}] \sim-1.4$ metallicity is found.

Since M31 and the Milky Way reveal so many differences in their evolutionary histories and environments (e.g., bulge luminosity, thin-disk scale, number, and types of globular clusters, scale sizes and types of satellite dwarf galaxies - e.g., Walterbos \& Kennicutt 1988; Barmby et al. 2000; Huxor et al. 2005; McConnachie \& Irwin 2006a, 2006b), it is of considerable interest that they seem to have developed similar metal-poor stellar components in their outskirts, likely near their formation epoch. If models of halo formation are correct (Bullock \& Johnston 2005; Renda et al. 2005; Font et al. 2006), this similarity in metallicity and dispersion, along with the similar dark matter masses (Evans \& Wilkinson 2000; Ibata et al. 2004), suggests that both galaxies must have originated in comparable overdensity contrasts and perhaps attracted similar numbers and masses of merger accretions early on.

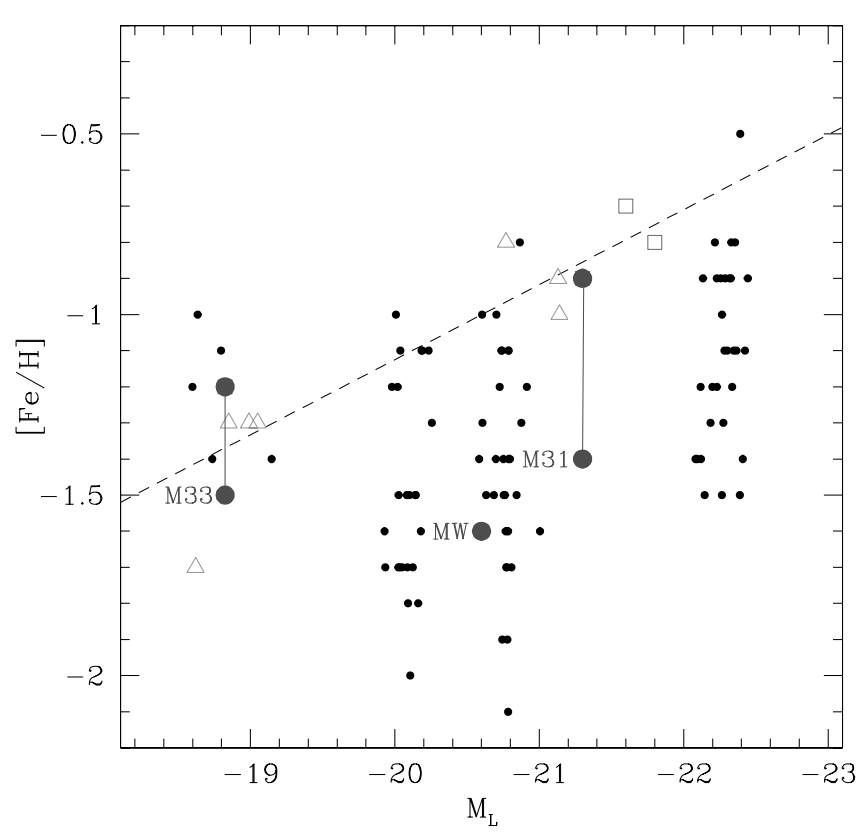

FIG. 12.-Stallar halo metallicity-total galactic $V$-band luminosity relation. Our kinematically selected metallicity measurements for M31 (this work) and for M33 (McConnachie et al. 2006) are shown as revised points connected by lines to literature values. Open triangles represent the HST data on other spiral galaxies from Mouhcine et al. (2005); open squares represent additional literature points extracted from Renda et al. (2005). Filled squares correspond to the peak of the metallicity distribution function (MDF) in halo simulations of Renda et al. (2005), where $68 \%$ of stellar particles in the MDF are enclosed within \pm 0.7 dex. The ensembles at different luminosity ranges represent all the stellar particles in the simulation at a projected distance $R>15 \mathrm{kpc}$, and corresponding to total masses (including dark matter) of $1 \times 10^{11}, 5 \times 10^{11}, 1 \times 10^{12}$, and $5 \times 10^{12} M \odot$, respectively. [See the electronic edition of the Journal for a color version of this figure.]

Our finding of a low halo metallicity in M31 contrasts, however, with recent measurements of the stellar halo metallicity distribution in a sample of more distant edge-on galaxies by Mouhcine et al. (2005). These authors derive photometric metallicity estimates from Hubble Space Telescope (HST) Wide Field Planetary Camera 2 (WFPC2) observations and find a strong correlation between halo [Fe/H] and galaxy luminosity (Fig. 12). For a luminosity similar to M31 or the Milky Way, their relation predicts a mean halo abundance of $[\mathrm{Fe} / \mathrm{H}] \sim-0.8$, significantly more metal-rich than that found here. It is important to point out that the fields studied by Mouhcine et al. (2005) are assumed to probe the stellar halo on the basis of their projected radii alone, but this assumption has not been verified by either structural decomposition or kinematics. We have shown that when halo stars are selected kinematically - arguably the best way in which to do so - then the metallicities inferred for the Milky Way and M31 are both similarily low. When combined with the recent discovery of metal-poor, $[\mathrm{Fe} / \mathrm{H}] \sim-1.5$, kinematically selected halo stars in the lower mass system M33 (McConnachie et al. 2006), this suggests that the true underlying stellar halo metallicity in spiral galaxies may have very little dependence on galaxy luminosity.

In Figure 12 we place our kinematically selected halo metallicity results for M31 and M33 in the distribution of Renda et al. (2005). Their results suggest that galaxies with metal-rich stellar halos at $z=0$ have a longer formation history, whereas galaxies with a more metal-poor stellar halo at $z=0$ have a shorter assembly, consistent with their halo [O/Fe] predictions. By kinematically selecting only the nonrotating halo components of the Local Group spirals, it is equivalent to selecting a more primordial halo in the Renda et al. (2005) simulations that would 
be isolated. However, Renda et al. (2005) define the halo as all the stellar particles in the simulation at a projected distance $R>$ $15 \mathrm{kpc}$. Clearly a direct application of this definition to M31 would result in an average $[\mathrm{Fe} / \mathrm{H}] \sim-1$, being drawn primarily from the dominant extended rotating component (I05). Note, however, that the Renda et al. (2005) simulations (Fig. 12) suggest that even defining the total halo in this way, finding a halo metallicity as rich as $[\mathrm{Fe} / \mathrm{H}] \sim-1$ is quite rare for virial masses as large as $\mathrm{M} 31\left(\sim 10^{12} M_{\odot}\right)$.

By contrast, in the Font et al. (2006) simulations finding an early assembled stellar halo with a $10^{12} M_{\odot}$ virial mass and an $[\mathrm{Fe} / \mathrm{H}] \sim-1.4$ (like the MW and M31) occurs in only $\sim 10 \%$ of the accretion histories, suggesting that the MW and M31 halos are actually quite rare for $\mathrm{L}^{*}$ spirals.

However, our findings should not be interpreted as claiming that all components of the $R<70 \mathrm{kpc}$ stellar halo are metalpoor, but simply that a nonrotating metal-poor component to M31 exists that is similar in $[\mathrm{Fe} / \mathrm{H}]$ to the halo in the Galaxy. There may also be a rotating halo subcomponent that is more metal-rich, which could effectively be windowed out of our current sample. Contamination from extended rotating component stars both in velocity and metallicity makes a detailed comparison difficult. Ferguson et al. (2002) and Irwin et al. (2005) noted that there is very clearly a persistent population with red colors (suggesting stars which may be metal-rich or young) along the minor axis, and beyond the region that I05 have characterized as the extended rotating component. While measuring the spectroscopic properties of this population would be interesting, the statistics in our minor-axis fields are currently insufficient to show any kinematic differences or even allow robust metallicity measurements for the red and blue RGBs. Future expansions of the data set may allow the characterization of this population.

Finally, it is of interest to understand how our new metal-poor component connects to the extended $R^{-2}$ halo component discovered photometrically along the minor axis by Irwin et al. (2005) and also discovered through spectroscopic confirmation of halolike, metal-poor stars at very large radius $(60-150 \mathrm{kpc}$ ) (Kalirai et al. 2006b; Gilbert et al. 2006). Are these all part of the same halo component, and is there a gradient in kinematics and metallicity? Again, it is difficult to assess, although the fact that massive satellites can be more easily accreted on prograde orbits (contaminating the disk, but leaving the spherical halo intact), suggests that such an extension of the halo would be physically motivated.

Tentative evidence from this work and from Kalirai et al. (2006b) for a more heterogeneous outer halo component distinct from the more phase mixed inner halo component is broadly consistent with the expectations from theory (Bullock \& Johnston 2005).

\section{CONCLUSIONS}

We have presented evidence for a metal-poor, $[\mathrm{Fe} / \mathrm{H}]=-1.4$, $\sigma=0.2$ dex, stellar halo component in M31, by kinematically isolating 827 nonrotating stars from our survey of $\sim 10,000$ stars with radial velocities from Keck II/DEIMOS. The halo component is detectable at radii from $10 \mathrm{kpc}$ to $70 \mathrm{kpc}$, and underlies a more dominant extended rotating component.

This metal-poor halo component has no detectable metallicity gradient, and is consistent with an early and rapid formation period as suggested by the simulations of Renda et al. (2005). The more metal-rich stellar halo components observed with $H S T$ by Mouhcine et al. (2005) may be indicative of components accreted later over longer assembly times.

For the first time, our analysis has also allowed us to constrain the kinematic properties of the M31 halo. The windowed halo sample shows no evidence for rotation about any axis, although there is clearly a drop in the velocity dispersion of the population with distance away from the galaxy center. Fitting an NFW model to these data and using the cosmologically motivated constraint that the model concentration $c<21$, provides a lower limit to the virial mass of the halo of $\mathrm{M}_{V}>9.0 \times 10^{11} M_{\odot}(99 \%$ confidence).

S. C. C. acknowledges support from NASA. G. F. L. acknowledges support through ARC DP0343508 and thanks the Australian Academy of Science for financial support in visiting the Institute of Astronomy, Cambridge. A. M. would like to thank J. Navarro and S. Ellison for financial support. Data presented herein were obtained using the W. M. Keck Observatory, which is operated as a scientific partnership among Caltech, the University of California, and NASA. The Observatory was made possible by the generous financial support of the W. M. Keck Foundation.
Abadi, M., Navarro, J., \& Steinmetz, M. 2006, MNRAS, 365, 747

Barmby, P., Huchra, J. P., Brodie, J. P., Forbes, D. A., Schroder, L. L., \& Grillmair, C. J. 2000, AJ, 119, 727

Bellazzini, M., et al. 2003, A\&A, 405, 867

Brown, T., et al. 2003, ApJ, 592, L17

Bullock, J. S., \& Johnston, K. V., ApJ, 2005, 635, 931

2004, in ASP Conf. Ser. 327, Satellites and Tidal Streams, ed. F. Prada,

D. Martinez-Delgado, \& T. Mahoney (San Francisco: ASP), 80

Bullock, J. S., Kravtsov, A. V., \& Weinberg, D. 2001, ApJ, 548, 33

Carretta, E., \& Gratton, R. 1997, A\&AS, 121, 95

Chapman, S. C., Ibata, R., Ferguson, A. M. N., Irwin, M., Lewis, G., McConnachie, A., \& Tanvir, N. 2005, ApJ, 632, 87L

Chapman, S. C., Ibata, R., Ferguson, A. M. N., Irwin, M., Lewis, G., \& Tanvir, N. 2006, ApJ, in press

Chiba, M., \& Beers, T. 2000, AJ, 119, 2843

2001, ApJ, 549, 325

Durrell, P., Harris, W., \& Pritchet, C. 1994, AJ, 108, 2114 2001, AJ, 121, 2557

Evans, N., \& Wilkinson, M. 2000, MNRAS, 316, 929

Faber, S. M., et al. 2003, Proc. SPIE, 4841, 1657

Ferguson, A., Irwin, M., Ibata, R., Lewis, G., \& Tanvir, N. 2002, AJ, 124, 1452

Font, A., Johnston, K. V., Bullock, J. S., \& Robertson, B, 2006, ApJ, 638, 585

Geehan, J. J., Fardal, M. A., Babul, A., \& Guhathakurta, P. 2006, MNRAS, 336,966

\section{REFERENCES}

Gilbert, K., et al. 2006, ApJ, submitted

Guhathakurta, P., et al. 2006, ApJ, in press

Helmi, A., \& White, S. D. M. 1999a, MNRAS, 307, 495

Holland, S., Fahlman, G. G., \& Richer, H. B. 1996, AJ, 112, 1035

Huxor, A., Tanvir, N. R., Irwin, M. J., Ibata, R., Collett, J. L., Ferguson, A. M. N., Bridges, T., \& Lewis, G. F. 2005, MNRAS, 360, 1007

Ibata, R., Chapman, S. C., Ferguson, A. M. N., Irwin, M., Lewis, G., \& McConnachie, A. 2004, MNRAS, 351, L94

Ibata, R., Chapman, S. C., Ferguson, A. M. N., Irwin, M., Lewis, G., \& Tanvir, N. 2005, ApJ, 634, 287 (I05)

Ibata, R. A., Gilmore, G., \& Irwin, M. J. 1994, Nature, 370, 194

Ibata, R., Irwin, M., Lewis, G., Ferguson, A., \& Tanvir, N. 2001a, Nature, 412, 49

Ibata, R., Lewis, G., Irwin, M., Totten, E., \& Quinn, T. 2001b, ApJ, 551, 294

Irwin, M. J., Ferguson, A. M. N., Ibata, R. A., Lewis, G. F., \& Tanvir, N. R. 2005, ApJ, 628, L105

Johnston, K. V., Sigurdsson, S., \& Hernquist, L. 1999, MNRAS, 302, 771

Kalirai, J. S., Guhathakurta, P., Majewski, S. R., Ostheimer, J. C., Rich, R., Michael, C., Reizel, D. B., \& Patterson, R. J. 2006a, ApJ, 641, 268

Kalirai, J. S., et al. 2006b, ApJ, 648, 389

Klypin, A., Zhao, H., \& Sommerville, R. 2002, ApJ, 573, 597

Lokas, E., \& Mamon, G. 2001, MNRAS, 321, 155

Martin, N., Ibata, R. A., Irwin, M. J., Chapman, S., Lewis, G. F., Ferguson, A. M. N., Taniver, N., \& McConnachie, A. W. 2006, MNRAS, MNRAS, 367, L69 
McConnachie, A. W., \& Irwin, M. J. 2006a, MNRAS, 365, 1263 2006b, MNRAS, 365, 902

McConnachie, A. W., Chapman, S. C., Ibata, R., Ferguson, A., Irwin, M. J., Lewis, G. F., \& Tanvir, N. 2006, ApJ, 647, L 25

McConnachie, A. W., Irwin, M., Ferguson, A., Ibata, R., Lewis, G. F., \& Tanvir, N. 2005, MNRAS, 356, 979

McConnachie, A., Irwin, M., Ibata, R., Ferguson, A., Lewis, G., \& Tanvir, N. 2003, MNRAS, 343, 1335

McConnachie, A., et al. 2004, MNRAS, 351, 94L

Mould, J., \& Kristian, J. 1986, ApJ, 305, 591

Mouhcine, M., Ferguson, H., Rich, M., Brown, T., \& Smith, T. 2005, ApJ, 633, 828

Navarro, J., Frenk, C., \& White, S, 1997, ApJ, 490, 493 (NFW)
Newberg, H. J., et al. 2002, ApJ, 569, 245

Penarrubia, J., McConnachie, A., \& Babul, A. 2006, MNRAS, ApJ, 650, L33

Pritchet, C., \& van den Bergh, S. 1988, ApJ, 331, 135

Reitzel, D. B., \& Guhathakurta, P. 2002, AJ, 124, 234

Reitzel, D. B., Guhathakurta, P., \& Rich, M. 2004, AJ, 127, 2133

Renda, A., Gibson, B., Mouhcine, M., Ibata, R., Kawata, D., Flynn, C., \& Brook, C. 2005, MNRAS, 363, 16L

Robin, A., Reylé, C., Derrière, S., \& Picaud, S. 2004, A\&A, 416, 157

Rutledge, G., Hesser, J., \& Stetson, P. 1997, PASP, 109, 883

Walterbos, R., \& Kennicutt, R. C., Jr. 1988, A\&A, 198, 61

White, S. D. M. \& Rees, M. J. 1978, MNRAS, 183, 341

Worthey, G., España, A., MacArthur, L. A., \& Courteau, S. 2005, ApJ, 631, 820 